\title{
Signatures of the Schwinger mechanism assisted by a fast-oscillating electric field
}

\author{
Selym Villalba-Chávez ${ }^{*}$ and Carsten Müller \\ Institut für Theoretische Physik, Heinrich-Heine-Universität Düsseldorf, \\ Universitätsstr. 1, 40225 Düsseldorf, Germany
}

(Received 15 July 2019; published 20 December 2019)

\begin{abstract}
The spontaneous production of electron-positron pairs from the vacuum-in a field configuration composed of a high-frequency electric mode of weak intensity and a strong constant electric field-is investigated. Asymptotic expressions for the single-particle distribution function ruling this nonperturbative process are established by considering the low-density approximation in the Boltzmann-Vlasov equation. An analytical formula for the density rate of yielded particles is established and is shown to manifest a nonperturbative dependence on both the strong and weak electric fields and to generalize previous findings by interpolating between the tunneling and multiphoton regimes. It is shown that-under appropriate circumstances - the created plasma of electrons and positrons might reach densities for which their recombinations into high-energy photons occurs copiously. On the basis of this feature, an experimental setup for observing the dynamically assisted Schwinger effect is put forward.
\end{abstract}

DOI: 10.1103/PhysRevD.100.116018

\section{INTRODUCTION}

Finding suitable and controllable experimental conditions to materialize the all-permeating quantum vacuum fluctuations has been a fundamental goal in particle physics since the time when our inert vacuum perception strictly changed into a nontrivial regulatory void, responsible for mediating the interactions between elementary particles. Even before the full establishment of QED, it was noted that this sort of vacuum instability could be conceived by producing electron-positron pairs if a macroscopic electric field $E$ is held in vacuum [1-3] (for a recent review, see Ref. [4]). Notwithstanding, the corresponding pair-production $(\mathrm{PP})$ rate $\mathcal{R} \sim \exp \left[-\pi E_{\mathrm{cr}} / E\right]$ provides evidence that an experimental verification of this so-called Schwinger mechanism is far from our reach, unless yet unaccessible field strengths-comparable to the critical scale of QED $E_{\mathrm{cr}}=m^{2} / e \sim 10^{16} \mathrm{~V} / \mathrm{cm}$ - become available. ${ }^{1}$ Although significant progresses toward high-intensity laser technology are raising our hopes of reaching the required field

\footnotetext{
*selym@tp1.uni-duesseldorf.de

${ }^{1}$ Here and henceforth, the mass and the absolute charge of an electron will be denoted by $m$ and $e$, respectively. Besides, throughout the manuscript, Heaviside-Lorentz units-with the speed of light and the Planck constant set to unity $c=\hbar=1$-are used.

Published by the American Physical Society under the terms of the Creative Commons Attribution 4.0 International license. Further distribution of this work must maintain attribution to the author(s) and the published article's title, journal citation, and DOI. Funded by SCOAP ${ }^{3}$.
}

strengths within the focal spot of multipetawatt laser pulses, it is rather likely that an experimental verification of the Schwinger PP process remains a challenging task to achieve, at least in a near future. This is because the expected peak field strengths of order $10^{-2} E_{\text {cr }}$ at the new generation of laser systems, including the Extreme Light Infrastructure (ELI) [5] and the Exawatt Center for Extreme Light Studies (XCELS) [6], would keep the production rate very small.

A central aspect in investigations aiming to relieve the exponential suppression of $\mathcal{R}$ is the identification of field setups, which may allow us to maximize the Schwinger effect [7-13]. Perhaps the most robust configuration found so far is the one implemented in what is nowadays known as the dynamically assisted Schwinger mechanism [14,15], where - in addition to a strong quasistatic electric field-a weak but high-frequency field component is superimposed. In the original papers on the subject, the combined field was composed of two Sauter pulses [14] or a constant electric field and a high-energy electromagnetic wave with $\omega \lesssim 2 m$ [15]. The latter ingredient-partially motivated by the experimental verification of the nonlinear BreitWheeler reaction [16] — stimulates the creation of pairs substantially. Indeed, first estimates resulting from this assisted scenario predict an enhancement of the PP rate $\mathcal{R} \sim \exp \left[-\kappa E_{\text {cr }} / E\right]$ with $0<\kappa \ll 1$, while its nonperturbative feature in the strong field strength is kept. Similar improvement has been predicted to take place in production channels other than the one described so far, provided the assisted high-frequency laser wave is present [17-19]. Qualitatively, this sort of catalysis is understood as a direct 
consequence of the absorption of photons from the weak field, which causes an effective reduction of the barrier width that an electron has to tunnel from negative to positive Dirac continuum. A large number of transitions are thus facilitated-pairs are created copiously-leading to increasing our chances for observing a signature of the vacuum instability.

This paper is devoted to study the spontaneous production of electron-positron pairs as might occur in a dynamically assisted setup driven by the combination of a constant and a purely time-dependent electric field. Our theoretical approach relies on the quantum transport equation that dictates the time evolution of the PP process [20-23]. It is noteworthy that several investigations of this nature have already been carried out, most of them by using numerical techniques from which valuable information and features have been extracted [24-32]. Meanwhile various researches have focused on deriving formulae for the created particle spectra [33-44]; this way crucial aspects are illuminated from which an optimized version of the aforementioned enhancement could be reached. Two recent papers went a step further by providing analytical expressions for the total probability of produced pairs $[45,46]$. In these investigations, a pertubative treatment in the weak field was used within the Wentzel Kramers Brillouin (WKB) and the worldline instanton methods, respectively. Particular attention was laid on weak fields with Sauter and Gaussian profiles. However, the case of a periodically oscillating mode was touched on only briefly. Here, we move a step further in these analytical studies by using a quantum kinetic approach in which both the weak and strong fields are treated nonperturbatively. We discuss in detail the case in which the assisted mode oscillates periodically and obtain a formula for the density rate of yielded particles which is shown to manifest a nonperturbative dependence on both the strong and weak electric fields and to interpolate between the tunneling and multiphoton regimes [see Eq. (22)]. Our expression generalizes the corresponding result in Ref. [45], which can be inferred from it by taking a suitable asymptotic limit. Besides, our formula allows us to understand the dependencies of the pair creation rate found by numerical means [27] and might eventually help — at the level of experiment - to elucidate the production signal among undesirable noises.

The outcomes of this analysis are exploited to reveal fundamental properties linked to the unstable nature of the yielded electron-positron plasma. Particular attention is paid to establish a formula for its lifetime. We show that this timescale decreases as the produced particle rate grows. As a consequence, the enhancement induced by the superposition of the fast-oscillating wave onto the strong field background does not translate necessarily into a beneficial aspect for a direct experimental detection of the spontaneous production of pairs from the vacuum. In light of this, we put forward a setup which aims to verify indirectly the realization of the dynamically assisted Schwinger effect. It relies on the detection of gamma photons, copiously emitted as a result of pair recombinations once the background has been switched off, i.e., from the annihilation of the residual plasma of electrons and positrons. The plausible signalization of the standard Schwinger mechanism through radiation channels has been subject to intense investigation. This idea was launched originally in Ref. [47] and further developed in a series of papers [48-51], most of them relying on the emergence of single-photon electron-positron annihilation processes taking place in the presence of the strong laser pulse. ${ }^{2}$ Improvements on this line of research have been reported recently in Ref. [61]. We complement these investigations by studying annihilation of the created plasma of electrons and positrons which remain after the external field has been switched off. In case of the unassisted Schwinger process, this yielded electron-positron plasma has not been in the focus of interest because it typically contains a very small amount of particles only. However, in the present situation of the assisted Schwinger mechanism, the residual plasma can be abundant, rendering an analysis of its dynamical evolution with annihilation into photons relevant. Consideration of the plasma evolution in the absence of the field moreover has the advantage here that no background of high-frequency photons, which have triggered the assisted PP and from which the recombination photons must be discriminated, is present anymore.

This paper is organized as follows. In Sec. II, we adopt the model to be analyzed and briefly summarize the main aspects linked to the quantum Vlasov equation and its solution within the low-density limit. The aforementioned approximation is particularized in Sec. III to the case in which the external background combines a strong static electric field and a fastoscillating electric field. There, we establish an analytic expression for the single-particle distribution function and discuss its behavior in various regimes of interest. Later on, in Sec. IV, we derive a compact asymptotic formula for the PP rate and reveal explicitly how the enhancement caused by the weak mode is closely connected with the perturbative PP rate associated with the absorption of photons. Details about some special aspects of these calculations are given in the Appendixes A and B. Finally, in Sec. V, we investigate the evolution of the electron-positron plasma after its creation via the assisted Schwinger mechanism. An expression for the total number of photons resulting from the annihilation of pairs at early times is derived. Some insight on the mentioned

\footnotetext{
${ }^{2}$ Generally speaking, in the field of the pulse, the theory admits two independent one-photon radiation channels linked to the tadpole and the field-dressed vertex, respectively. The study of the former has also been exploited to reveal valuable insights in other yet unobserved nonlinear QED phenomena. See, for instance, Refs. [52-55]. The pair annihilation occurring during the time when the field is on was first investigated numerically in Ref. [56]. Likewise, the electron-positron recombination driven by a plane wave has been studied in Refs. [57-59]; see also Ref. [60].
} 
experimental setup aiming to detect these photons, and thus the spontaneous production of pairs, is given afterward.

\section{GENERAL ASPECTS}

We consider the spontaneous production of electronpositron pairs taking place in a time-dependent but homogeneous electric field combining a strong and a weak mode with frequencies $\Omega$ and $\omega$, respectively. We will suppose this field is localized temporally between $-T / 2 \leq t \leq T / 2$, its pulse length $T=2 \pi N / \omega$ being determined by the number of cycles $N$ and $\omega$. In the following, we further restrict the model to the case in which the variation linked to the perturbative mode is much faster than the one undergone by the strong field counterpart $(\omega \gg \Omega)$ and where, accordingly, $N$ is very large. Hence, the PP problem can be formulated as if the creation process were taking place in a background characterized by a constant electric field and a fast-oscillating mode, generated by the four-potential

$$
\mathcal{A}^{\mu}(t)=-\left[E_{s} t+\frac{E_{w}}{\omega} \sin (\omega t)\right] b^{\mu} \Theta(t+T / 2) \Theta(T / 2-t),
$$

where $b^{\mu}=(0,0,1,0)$ is the polarization four-vector and $\Theta(x)$ denotes the unit step function: $\Theta(x)=1$ at $x \geq 0$ and $\Theta(x)=0$ at $x<0$. Here, the subscripts " $s$ " and " $w$ " are used to identify the strong and weak field strengths $\left(E_{w} \ll E_{s}\right)$, respectively.

Our investigation adopts the quantum kinetic approach as a theoretical tool to describe the production of electronpositron pairs. This formulation-which is equivalent to other well-known approaches based on QED in unstable vacuum $[62,63]$ - comprises the dynamical information of the PP process in the single-particle distribution function $W(\boldsymbol{p} ; t)$ - summed over the spin projections-of electrons and positrons to which the degrees of freedom in the external field are relaxed at asymptotically large times $(t \rightarrow \pm \infty)$, i.e., when the electric field is switched off $\boldsymbol{E}( \pm \infty) \rightarrow 0$. The time evolution of this quantity is dictated by a quantum Boltzmann-Vlasov equation [20-23], the integrodifferential version

$$
\begin{aligned}
\dot{W}(\boldsymbol{p} ; t)= & Q(\boldsymbol{p}, t) \int_{-\infty}^{t} d \tilde{t} Q(\boldsymbol{p}, \tilde{t}) \\
& \times[1-W(\boldsymbol{p} ; \tilde{t})] \cos \left[2 \int_{\tilde{t}}^{t} d t^{\prime} w_{\boldsymbol{p}}\left(t^{\prime}\right)\right]
\end{aligned}
$$

of which manifests both the nonequilibrium nature of the PP process and its non-Markovian feature. ${ }^{3}$ The formula above assumes the vacuum initial condition $W(\boldsymbol{p},-\infty)=0$

\footnotetext{
${ }^{3}$ The quantum field theoretical approach to the pair-production problem - as encompassed by Eq. (2) concisely - can be formulated alternatively through a Riccati equation $[64,65]$ or via a representation involving three coupled ordinary differential equations. See, for instance, Refs. [34,37,66].
}

and applies the notation $\dot{W}(\boldsymbol{p} ; t) \equiv \partial W(\boldsymbol{p} ; t) / \partial t$. Besides, it is characterized by the function $Q(\boldsymbol{p}, t) \equiv e E(t) \epsilon_{\perp} / w_{p}^{2}(t)$, which depends on the transverse energy of the Dirac fermions $\epsilon_{\perp}=\sqrt{m^{2}+p_{\perp}^{2}}$ and the respective total energy squared $w_{p}^{2}(t)=\epsilon_{\perp}^{2}+\left[p_{\|}-e \mathcal{A}(t)\right]^{2}$ of a positron. Here, $\boldsymbol{p}_{\perp}=\left(p_{x}, 0, p_{z}\right)$ and $\boldsymbol{p}_{\|}=\left(0, p_{y}, 0\right)$ are the components of the canonical momentum perpendicular and parallel to the direction of $\boldsymbol{E}(t)$, respectively.

It is known that Eq. (2) can only be solved exactly for a few special backgrounds, e.g., constant and Sauter-type electric fields. Finding analytic solutions beyond the aforementioned configurations is a difficult task. However, estimates can be obtained by using the low-density approximation $[W(\boldsymbol{p} ; t) \ll 1]$ within the Boltzmann-Vlasov equation. In such a case, the single-particle distribution function at times, for which the field has been switched off $\left[W_{T}(\boldsymbol{p}) \equiv \lim _{t \rightarrow T} W(\boldsymbol{p} ; t)\right]$, can be approximated by $[9,22]$

$$
W_{T}(\boldsymbol{p}) \approx \frac{1}{2}\left|\int_{-T / 2}^{T / 2} d \tilde{t} Q_{p}(\tilde{t}) e^{i \Lambda_{p}(\tilde{t})}\right|^{2}
$$

with $\Lambda_{p}(\tilde{t}) \equiv 2 \int_{0}^{\tilde{t}} d t w_{p}(t)$. We note that the integration contained in this formula is nothing but the solution of the linearized Riccati equation on which the study in Ref. [65] relies. At this point, it turns out to be rather illuminating to perform the change of variables $\tau=\left[p_{\|}-e \mathcal{A}(\tilde{t})\right] / \epsilon_{\perp}$ and $\tilde{\tau}=\left[p_{\|}-e \mathcal{A}(t)\right] / \epsilon_{\perp}$. As a consequence, the integral in Eq. (3) becomes

$$
\begin{aligned}
\int_{-T / 2}^{T / 2} d \tilde{t} \ldots & =\int_{\frac{\gamma_{\Perp}}{\gamma_{\perp}}-\frac{\pi N}{\gamma_{\perp}}}^{\frac{\gamma_{\Perp}}{\gamma_{\perp}}+\frac{\pi N}{\gamma_{1}}} \frac{d \tau}{1+\tau^{2}} \exp \left[\frac{\epsilon_{\perp}^{2}}{e E_{S}} \mathcal{S}(\tau)\right] \\
\mathcal{S}(\tau) & =2 i \int_{0}^{\tau} d \tilde{\tau} \frac{\left(1+\tilde{\tau}^{2}\right)^{1 / 2}}{1+\varepsilon \cos (\omega t)}
\end{aligned}
$$

where $\varepsilon=E_{w} / E_{s} \ll 1$ parametrizes the relative weakness of the fast-oscillating mode. The expression above constitutes the starting point for further considerations. In its second line, $t$ has to be considered as a function of $\tilde{\tau}$. However, this inversion cannot be determined analytically, but only through reversion of the corresponding series $[67,68]$. In this case, the leading-order term

$$
t(\tilde{\tau}) \approx \frac{1}{\omega}\left(\gamma_{\perp} \tilde{\tau}-\gamma_{\|}\right)
$$

coincides with the inverse of the function $\tau(t)$ averaged over a cycle of the weak field. Here, we have introduced the dimensionless parameters

$$
\gamma_{\|}=\gamma \frac{p_{\|}}{m} \quad \text { and } \quad \gamma_{\perp}=\gamma \frac{\epsilon_{\perp}}{m} .
$$

Observe that, in the limit of $p_{\perp} \rightarrow 0, \gamma_{\perp}$ reduces to the combined Keldysh parameter $\left[\gamma=\omega m /\left(e E_{s}\right)\right]$. In order to 
suitably fit the external parameters to current and foreseeable experimental setups, we will suppose hereafter that $m^{2} \gg\left(e E_{s}\right)$ and $2 m>\omega$. We note that an assisted scenario with $2 m>\omega \gtrsim m^{4}$ is characterized by the restriction $\gamma_{\perp} \geq \gamma \gg 1$. Conversely, if $\omega \ll m^{3} /\left(e E_{s}\right)$-leading to $\gamma \ll 1$-the effective reduction of the barrier width between the negative and positive continuum is expected to be almost insignificant, and the oscillating field would not play a significant role in the production of pairs.

\section{PROPERTIES OF THE PARTICLE SPECTRUM}

We wish to find closed-form analytic expressions for Eq. (3) in the case characterized by the condition $\pi N \gg \gamma_{\perp}$, $\left|\gamma_{\|}\right|$. Therefore, the treatment developed in this subsection is limited to small momentum components relative to the one associated with the external field, i.e., to values $\left|p_{\|}\right| \ll$ $e E_{s} T / 2$ and $\left|p_{\perp}\right| \ll e E_{s} T / 2$. To facilitate the mathematical treatment of the problem, we will formally extend the outer integration limits in Eq. (4) to $\pm \infty$. As a consequence, the single-particle distribution function $W_{T}(\boldsymbol{p})$ [see Eq. (3)] approaches to a $2 \pi$-periodic function in $\gamma_{\|}$. Correspondingly, its dependence on this variable will be investigated in the interval $-\pi<\gamma_{\|} \leq \pi$.

Since the factor $\epsilon_{\perp}^{2} /\left(e E_{s}\right) \geq m^{2} /\left(e E_{s}\right) \gg 1$, the exponential in Eq. (4) oscillates very fast, and the steepestdecent method represents a suitable tool to carry out its outer integration. In order to apply this method, we first extend the integration variables to the complex $\tau$ plane. As it is characteristic in problems of this nature, the poles at $\tau= \pm i$ are also branch points of the integrand. The branch cuts are then chosen from $\tau=i$ to $\tau=i \infty$ and from $\tau=-i$ to $\tau=-i \infty$, i.e., $\tau^{2}+1=(\tau+i)(\tau-i)=\mid \tau+i \| \tau-$ $i \mid e^{i \varphi_{+}} e^{i \varphi_{-}}$with $-3 \pi / 2 \leq \varphi_{+}<\pi / 2$ and $-\pi / 2 \leq \varphi_{-}<$ $3 \pi / 2$ referring to the local polar angle linked to $\tau=+i$ and $\tau=-i$, respectively. Still, in the cut $\tau$ plane, there exist poles linked to the integrand of $\mathcal{S}(\tau)$,

$$
\tau_{ \pm \nprec}=\frac{\gamma_{\|}+(2 k-1) \pi}{\gamma_{\perp}} \pm i \frac{\gamma_{\mathrm{cr}}}{\gamma_{\perp}}, \quad k \in \mathbb{Z}
$$

where a loss of analyticity is exhibited. While the integer value $k$ manifests the periodicity in $\gamma_{\|}$, the critical Keldysh parameter $[36,45,46,70]$

$$
\gamma_{\mathrm{cr}}=\ln \left(\frac{2}{\varepsilon}\right), \quad \varepsilon \ll 1
$$

rules two different scenarios depending on whether $\gamma_{\perp}$ does or does not exceed the value of $\gamma_{\mathrm{cr}}$. Below, we will further describe this point.

\footnotetext{
${ }^{4}$ In connection with PP in this frequency regime, see Refs. [27,69].
}

Now, the saddle points linked to the exponent $(\tau= \pm i)$ coincide with the branch points found previously. In a vicinity of $\tau=i$, the preexponential of the integrand behaves as $1 /[2 i(\tau-i)]$, whereas

$$
\begin{aligned}
\mathcal{S}(\tau) & \approx \frac{i}{2} \mathcal{S}_{0}+\frac{1}{\sqrt{2}}|\tau-i|^{3 / 2}|\kappa| e^{i \arg \kappa+i \frac{3 \pi}{4}+i \frac{3}{2} \varphi_{+}}, \\
\mathcal{S}_{0} & =4 \int_{0}^{i} d \tilde{\tau} \frac{\left(1+\tilde{\tau}^{2}\right)^{1 / 2}}{1+\varepsilon \cos \left(\gamma_{\perp} \tilde{\tau}-\gamma_{\|}\right)} .
\end{aligned}
$$

In the first line, $\arg \kappa$ denotes the principal value of the argument of $\kappa=[1+\varepsilon \cos (\omega t(i))]^{-1}$. Here, the directions of the steepest descents can be locally approximated by choosing $\varphi_{+}=-\frac{\pi}{2}+\frac{2}{3} k \pi-\frac{2}{3} \arg \kappa$, with $k \in \mathbb{Z}$, such that the condition $\cos \left(\arg \kappa+\frac{3}{4} \pi+\frac{3}{2} \varphi_{+}\right)<0$ holds. In connection, we find

$$
\varphi_{+1}=\frac{\pi}{6}-\frac{2}{3} \arg \kappa, \quad \varphi_{+2}=-\frac{7 \pi}{6}-\frac{2}{3} \arg \kappa .
$$

Since the angles above must lie within the interval $\left[-\frac{3 \pi}{2}, \frac{\pi}{2}\right)$ [read above Eq. (9)], arg $\kappa$ turns out to be restricted to the region $-\frac{\pi}{2}<\arg \kappa \leq \frac{\pi}{2}$, with

$$
\arg \kappa=\tan ^{-1}\left[\frac{\varepsilon \sinh \left(\gamma_{\perp}\right) \sin \left(\gamma_{\|}\right)}{1+\varepsilon \cosh \left(\gamma_{\perp}\right) \cos \left(\gamma_{\|}\right)}\right]+s_{i}
$$

with $s_{1,4}=0, s_{2}=\pi, s_{3}=-\pi$, where the choice of the constant $s_{i}$ corresponds to suitable values in each of the four quadrants [71]. Manifestly, Eq. (11) reveals that the directions of the steepest descents change as $\gamma_{\perp}$ and $\gamma_{\|}$are varied. We remark that, in the absence of the perturbative mode $(\varepsilon=0 \Leftrightarrow \arg \kappa=0)$, these directions are fully specified: $\varphi_{+1}=\pi / 6$ and $\varphi_{+2}=-7 \pi / 6$. It is worth remarking that these angles do not change appreciably when the fast oscillating field is turned on $(\varepsilon \neq 0)$ and $\gamma_{\mathrm{cr}} \gg \gamma_{\perp}$, no matter the value of $\gamma_{\|}$. The situation does not change either if $\varepsilon \neq 0, \gamma_{\perp} \gg \gamma_{\text {cr }}$ with $\gamma_{\perp} \gg 1$ and $\left|\gamma_{\|}\right| \ll 1$. Even for $\left|\gamma_{\|}\right| \ll 1$ and $\gamma_{\perp} \sim \gamma_{\text {cr }}>2$, it can be verified that $\arg \kappa \approx 0$, and the directions of the steepest descents approach locally to those arising when the strong electric field is present only.

We take advantage of the described feature to deform the initial integration contour [see Eq. (4)], as depicted in Fig. 1. The portion covering the real axis deviates several times through circles $c_{\kappa}$ with an infinitesimal radius $(\mu \rightarrow 0)$. At this point, it should be understood that each of them joins the real $\tau$ axis via parallel shortcuts with opposite directions. However, in the picture, they have been omitted for simplicity and because they do not contribute at all. Here, $\ell$ labels a circumvented pole between the most distant extremes of the circuit [see Eq. (7)]. In the picture, the imaginary parts of the poles have been taken with $\gamma_{\perp} \gg \gamma_{\mathrm{cr}}$. For $\gamma_{\perp} \ll \gamma_{\mathrm{cr}}$, the pole locations are moved 


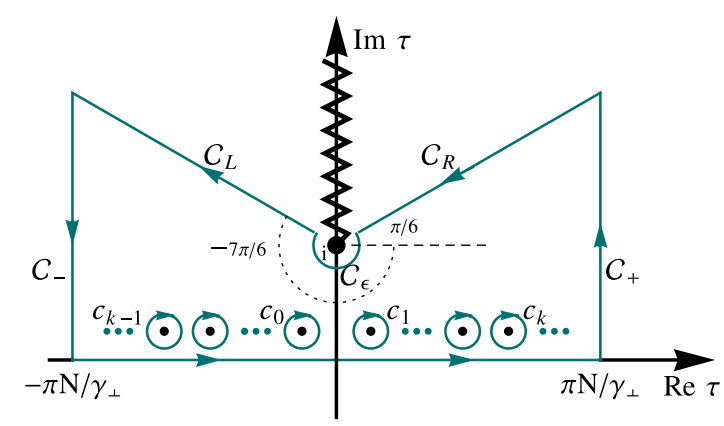

FIG. 1. Integration contour in the complex plane $\tau$ for $\arg \kappa \approx 0$. The positive branch cut, defined from $\tau=i$ to $\tau=i \infty$, is depicted by a vertical zigzag line. Here, the imaginary parts of the poles have been taken smaller than unity $\left(\gamma_{\mathrm{cr}} \ll \gamma_{\perp}\right)$. Shortcuts joining each circumventing circle with the real $\tau$ axis have been omitted for simplicity. They give no contribution as, for each circle, both lie infinitesimally close together and have opposite orientation.

upward, and many of them could lie above the path $\Gamma=\mathcal{C}_{\mathcal{R}} \cup \mathcal{C}_{\epsilon} \cup \mathcal{C}_{\mathcal{L}}$. In such a situation, the contour of integration is chosen similarly to the previous case: those poles remaining below $\Gamma$ are then eluded. Consequently, Cauchy's theorem allows us to express

$$
\begin{aligned}
\int_{-\infty}^{\infty} d \tau \ldots= & -\exp \left[\frac{i \epsilon_{\perp}^{2}}{2 e E_{s}} \mathcal{S}_{0}\right] \\
& \times \int_{\Gamma} \frac{d \tau}{2 i(\tau-i)} \exp \left[\frac{\epsilon_{\perp}^{2}}{\sqrt{2} e E_{s}} \kappa[i(\tau-i)]^{3 / 2}\right],
\end{aligned}
$$

where we have taken into account that the contribution linked to each pole's circumvention, i.e., over $c_{k}$, vanishes as $r \rightarrow 0$ (for details, read Appendix A). Likewise, we have considered that the integrations over $\mathcal{C}_{ \pm}$give no contributions when $N \rightarrow \infty$. The details of this considerations are summarized in Appendix B.

We point out that Eq. (12) can also be applied to those cases in which the integration contour differs substantially from the one analyzed explicitly here, provided no contribution arises from those circuits connecting the region $-\pi N / \gamma_{\perp} \leq \operatorname{Re} \tau \leq \pi N / \gamma_{\perp}$ with the sectors ending in the steepest descents. Hereafter, we will suppose that this is the case. Thus, by performing the map $w=\epsilon_{\perp}^{2} \kappa[i(\tau-i)]^{3 / 2} /\left[\sqrt{2} e E_{s}\right]$, Eq. (12) becomes

$$
\int_{-\infty}^{\infty} d \tau \ldots=\frac{2 \pi i}{3} \exp \left[\frac{i \epsilon_{\perp}^{2}}{2 e E_{s}} \mathcal{S}_{0}\right] \oint_{\tilde{\Gamma}} \frac{d w}{2 \pi w} e^{w}
$$

Observe that the closed path of integration $\tilde{\Gamma}$ (see Fig. 2) encloses a single pole at infinity $(w=+\infty)$. Hence, the application of the residue theorem leads to $\oint_{\Gamma} d w e^{w} /(2 \pi w)=i[72]$. We substitute this outcome into Eq. (13). The resulting expression is inserted into Eq. (3)

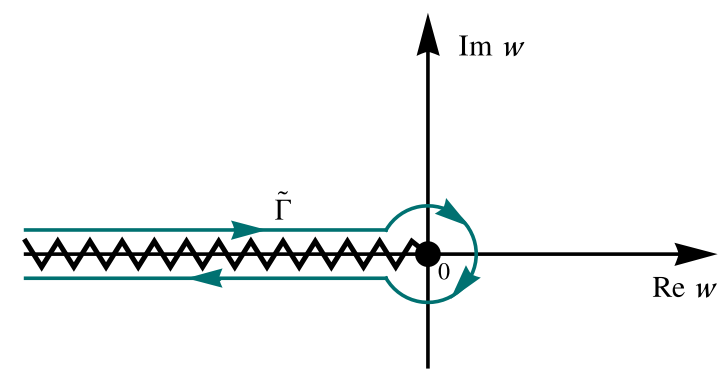

FIG. 2. Path of integration in the complex $w$ plane. Note that it encloses the outer region in counterclockwise sense.

afterward. As a consequence, the single-particle distribution function reduces to

$$
W_{T}(\boldsymbol{p}) \approx 2 e^{-\frac{\epsilon_{\perp}^{2}}{c E_{s}} \operatorname{Im} \mathcal{S}_{0}\left(\gamma_{\perp}, \gamma_{\|}\right)}
$$

where an unessential preexponential factor of the order of unity has been omitted $\left(\pi^{2} / 9 \approx 1.1\right)$. We stress that $\operatorname{Im} \mathcal{S}_{0}\left(\gamma_{\perp}, \gamma_{\|}\right)$is given in Eq. (9). By taking $\tau=i y$, it reads

$\operatorname{Im} \mathcal{S}_{0}\left(\gamma_{\perp}, \gamma_{\|}\right)=4 \int_{0}^{1} d y \frac{\sqrt{1-y^{2}} \cos ^{2}\left(\phi_{y}\right)}{1+\varepsilon \cosh \left(\gamma_{\perp} y\right) \cos \left(\gamma_{\|}\right)}$

Here, the function $\phi_{y}$ is the angle determined by the $\tan ^{-1}$ function involved in Eq. (11), with $\gamma_{\perp}$ replaced by $\gamma_{\perp} y$. We remark that the expression above is an even function in $\gamma_{\|}$. Hence, we further restrict our investigation to $0 \leq \gamma_{\|} \leq \pi$. It is worth noting that a similar expression for $W_{T}(\boldsymbol{p})$ can be inferred from the WKB-method. This fact provides an evidence that, although Eqs. (14) and (15) apply strictly in the regimes $\gamma_{\mathrm{cr}} \gg \gamma_{\perp}$ and $\gamma_{\perp} \gg \max \left\{1, \gamma_{\mathrm{cr}}\right\}$, they can be used as well to extrapolate the behavior of $W_{T}(\boldsymbol{p})$ to intermediate regions [see, for instance, Fig. 3 below].

Observe that, in the limit of $\varepsilon \rightarrow 0, \operatorname{Im} \mathcal{S}_{0}\left(\gamma_{\perp}, \gamma_{\|}\right)=\pi$. Hence, $W_{T}(\boldsymbol{p})$ reduces to the known expression in a constant electric field [66]: $W_{T}(\boldsymbol{p}) \approx 2 \exp \left[-\pi \epsilon_{\perp}^{2} /\left(e E_{\mathrm{cr}}\right)\right]$. The situation changes when $\varepsilon \neq 0$. To show this, we investigate some analytic and asymptotic properties of Eq. (15). First of all, when setting $\partial \operatorname{Im} \mathcal{S}_{0}\left(\gamma_{\perp}, \gamma_{\|}\right) / \partial \gamma_{\|}$to zero, we find that the corresponding extreme points are located at the borders of the region encompassed by $0 \leq \gamma_{\|} \leq \pi$. To elucidate which of them maximize and minimize Eq. (15), we will establish its asymptotic formulas evaluated at $\gamma_{\|}=0$ and $\gamma_{\|}=\pi$, respectively. Let us begin with the case in which $\gamma_{\perp} \gg \gamma_{\text {cr }}$ with $\gamma_{\perp} \gg 1$. We then introduce a positive splitting parameter $y_{0}$ satisfying the conditions $\gamma_{\perp}^{-1} \ll y_{0} \ll 1$ and $\gamma_{\text {cr }} / \gamma_{\perp} \ll y_{0}$. Afterward, the $y$ integration is divided as follows: $\operatorname{Im} \mathcal{S}_{0}\left(\gamma_{\perp}, \gamma_{\|}\right)=$ $4 \int_{0}^{y_{0}} d y \ldots+4 \int_{y_{0}}^{1} d y \ldots$. In the integral defined over the region $\left[0, y_{0}\right]$, the integration variable is very small $(y \ll 1)$, which allows us to approximate the square root contained in 
the integrand by unity $\left[\left(1-y^{2}\right)^{1 / 2} \approx 1\right]$. Conversely, the main contribution to the integral defined over $\left[y_{0}, 1\right]$ results from those values of $y$ fulfilling the condition $y \gg \gamma_{\mathrm{cr}} / \gamma_{\perp}$, in which case the integrand can be approximated by $\sim \pm 2 \exp \left[-\gamma_{\perp} y\right] / \varepsilon$. Consequently,

$$
\begin{aligned}
\operatorname{Im} \mathcal{S}_{0}\left(\gamma_{\perp}, \gamma_{\|}\right) \approx & 4 \int_{0}^{\infty} \frac{d y}{1 \pm \varepsilon \cosh \left(\gamma_{\perp} y\right)} \\
& \pm \frac{8}{\varepsilon} \int_{y_{0}}^{1} d y e^{-\gamma_{\perp} y} \sqrt{1-y^{2}} \\
& -4 \int_{y_{0}}^{\infty} \frac{d y}{1 \pm \varepsilon \cosh \left(\gamma_{\perp} y\right)} .
\end{aligned}
$$

While the positive sign corresponds to $\gamma_{\|}=0$, the negative one is associated with $\gamma_{\|}=\pi$. Now, the first integral involved in this expression can be calculated by using Eq. (3.513.2) in Ref. [73]. The remaining two can be combined in an integral independent of the parameter $y_{0}$. Indeed, note that, as $\gamma_{\perp} \gg 1$, the integrand in $\int_{y_{0}}^{1} d y \ldots$ behaves as approximately $\exp \left[\gamma_{\perp} y\right]$, whereas the one defined in $\left[y_{0}, \infty\right)$ can be approximated by $\pm 2 \exp \left[-\gamma_{\perp} y\right] / \varepsilon$. Keeping all these details in mind, we obtain

$$
\operatorname{Im} \mathcal{S}_{0}\left(\gamma_{\perp}, \gamma_{\|}\right) \approx 4 \begin{cases}\frac{\gamma_{\mathrm{cr}}}{\gamma_{\perp}}-\frac{1}{\gamma_{\perp}} e^{-\gamma_{\perp}} & \text { for } \gamma_{\|}=0, \\ \frac{\gamma_{\mathrm{cr}}}{\gamma_{\perp}}+\frac{1}{\gamma_{\perp}} e^{-\gamma_{\perp}} & \text { for } \gamma_{\|}=\pi .\end{cases}
$$

As the extreme points linked to $\gamma_{\|}$coincide with the borders of the interval $[0, \pi]$, and $\operatorname{Im} \mathcal{S}_{0}\left(\gamma_{\perp}, \pi\right)>$ $\operatorname{Im} \mathcal{S}_{0}\left(\gamma_{\perp}, 0\right)$, the quantity $\operatorname{Im} \mathcal{S}_{0}\left(\gamma_{\perp}, \gamma_{\|}\right)$as a function of $\gamma_{\|}$grows monotonically in this region for any $\gamma_{\perp}$ satisfying the conditions $\gamma_{\perp} \gg \gamma_{\text {cr }}$ and $\gamma_{\perp} \gg 1$. This behavior is also verified by looking at the solid curves in Fig. 3, which have been obtained by setting $\gamma_{\|}=0$ (black), $\gamma_{\|}=\pi / 2$ (red), and $\gamma_{\|}=\pi$ (darker cyan). Furthermore, the formula above reveals that the amplitude of oscillations in $\gamma_{\|}$decreases as $8 \exp \left[-\gamma_{\perp}\right] / \gamma_{\perp}$ when $\gamma_{\perp}$ grows, a fact exhibited clearly in Fig. 3 (compare the solid curves in black and darker cyan at $\gamma_{\perp}=10$, for instance). We remark that this trend changes, as the condition $\gamma_{\mathrm{cr}} \gg \gamma_{\perp}$ is fulfilled. Under this circumstance, all instances in Eq. (15) depending on $\varepsilon$ can be Taylor expanded. After the integration,

$$
\operatorname{Im} \mathcal{S}_{0}\left(\gamma_{\perp}, \gamma_{\|}\right) \approx \pi-\frac{2 \pi}{\gamma_{\perp}} \varepsilon \mathcal{I}_{1}\left(\gamma_{\perp}\right) \cos \left(\gamma_{\|}\right)
$$

where Eq. (3.534.1) in Ref. [73] has been used. Here, $\mathcal{I}_{1}(x)=\frac{x}{2}+\frac{x^{3}}{2^{2} 4}+\frac{x^{5}}{2^{2} 4^{2} 6}+\cdots$ denotes the modified Bessel function of the first kind with order 1 [74]. The formula above coincides with Eq. (37) in Ref. [36]. It manifests clearly a monotonic growing in $0 \leq \gamma_{\|} \leq \pi$. Furthermore, in the current regime, the amplitude of oscillation in $\gamma_{\|}$ scales as approximately $2 \pi \varepsilon$.

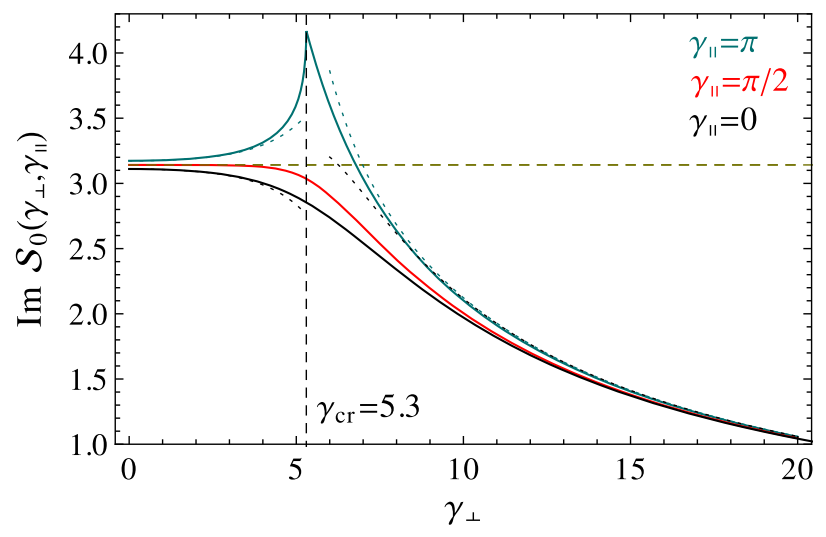

FIG. 3. Behavior of the function $\operatorname{Im} \mathcal{S}_{0}\left(\gamma_{\perp}, \gamma_{\|}\right)$as a function of $\gamma_{\perp}: \gamma_{\|}=0$ (black), $\gamma_{\|}=\pi / 2$ (red), and $\gamma_{\|}=\pi$ (darker cyan). The horizontal dashed line in olive corresponds to the value $\operatorname{Im} \mathcal{S}_{0}\left(\gamma_{\perp}, \gamma_{\|}\right)=\pi$ that results when the fast-oscillating wave is not present, whereas the asymptotic trends [Eqs. (18) and (17)] linked to the cases in which $\gamma_{\|}=0$ and $\gamma_{\|}=\pi$ are shown in dotted style. Here, the vertical dashed line indicates the critical value $\gamma_{\mathrm{cr}} \approx 5.3$ corresponding to $\varepsilon=10^{-2}$.

Interestingly, when $\gamma_{\perp}$ moves away from $\gamma_{\text {cr }}$ toward larger values, all solid curves in Fig. 3 show a significant falling as compared to the characteristic value linked to the constant field case $\left(\operatorname{Im} \mathcal{S}_{0} \approx \pi\right)$. Indeed, at $\gamma_{\perp}=20$, they have fallen to $\operatorname{Im} \mathcal{S}_{0} \approx 1$. Besides, as Eq. (17) applies for $\gamma_{\perp} \gg \gamma_{\text {cr }}$ with $\gamma_{\perp} \gg 1$, the exponential contributions can be safely ignored. This fact implies that $\operatorname{Im} \mathcal{S}_{0}\left(\gamma_{\perp}, \gamma_{\|}\right)$does not undergo an appreciable variation in $\gamma_{\|}$. Keeping all these details in mind, we find that the single-particle distribution function [see Eq. (14)] behaves as

$$
W_{T}(\boldsymbol{p}) \approx 2\left(\frac{\varepsilon}{2}\right)^{\frac{4 \varepsilon_{\perp}}{\omega}}, \quad \gamma_{\perp} \gg \max \left\{1, \gamma_{\mathrm{cr}}\right\}
$$

This expression deserves further comments. First, at $\boldsymbol{p}=0$, the exponent associated with this asymptotic formula coincides with the minimal "number" of quanta necessary to produce a pair at rest from the weak mode solely. This observation already provides evidence that an enhancement in $W_{T}(\boldsymbol{p})$ could take place via the absorption of a quantum from the fast-oscillating field, as compared with the case in which only a constant electric field drives the vacuum instability. Indeed, let us suppose the particles are created in an assisted field setup characterized by the following parameters: $\omega=1.7 \mathrm{~m}, E_{s}=10^{-1} E_{\mathrm{cr}}$, i.e., $\gamma \approx 17$, and $E_{w}=10^{-3} E_{\mathrm{cr}}$ corresponding to $\varepsilon=10^{-2}\left[\gamma_{\mathrm{cr}} \approx 5.3\right]$. Under such circumstances, we find that $W_{T}(\mathbf{0}) \sim 10^{-5}$ exceeds by 9 orders of magnitude the corresponding distribution function $W_{T}(\mathbf{0}) \sim 10^{-14}$ of the standard Schwinger mechanism. Apparently, the enhancement becomes stronger as $E_{s}$ decreases gradually. However, it is worth pointing out that such a trend is justified whenever $W_{T}(\boldsymbol{p})$ remains smaller than unity [read discussion above Eq. (3)]. 
Hence, at $\boldsymbol{p}=\mathbf{0}$, this condition translates into the restriction $E_{s} \gg \frac{1}{2} E_{w} 2^{\frac{\omega}{4 m}}$, and by using the parameters above, for instance, it will imply that $E_{s} \gg 7 \times 10^{-4} E_{\text {cr }}$.

\section{PAIR-PRODUCTION RATE}

The density of created electron-positron pairs follows from integration of the single-particle distribution function over the three momentum components. When exploiting the $2 \pi m \gamma^{-1}$-periodicity of $W_{T}(\boldsymbol{p})$ in $p_{\|}$[see below Eq. (15)], we can write

$$
\begin{aligned}
\mathcal{N} & =\int \frac{d^{3} p}{(2 \pi)^{3}} W_{T}(\boldsymbol{p}) \\
& =\frac{e E_{s} T}{2 \pi^{2}} \int_{0}^{\pi} d \gamma_{\|} \int_{0}^{\infty} \frac{d p_{\perp}}{2 \pi} p_{\perp} W_{T}(\boldsymbol{p}),
\end{aligned}
$$

where the even feature of $W_{T}(\boldsymbol{p})$ in $p_{\|}$has also been used. To satisfy the condition under which $W_{T}(\boldsymbol{p})$ was derived (see the first paragraph in Sec. III), the integral over $p_{\perp}$ must be performed over the region $\left[0, p_{\max }\right]$ with $p_{\max } \ll e E_{s} T$. However, the fast damping of its integrand in this variable allows us to extend its upper integration limit to infinity with no appreciable error. In contrast, due to the periodic feature of $W_{T}(\boldsymbol{p})$ in $p_{\|}$, the integration over this variable-originally covering the region $(-\infty, \infty)$ has been limited to the domain $\left|p_{\|}\right| \leqslant \frac{1}{2} e E_{s} T$.

At this point, it turns out to be convenient to carry out the change of variable $s^{2}=\omega^{2} \epsilon_{\perp}^{2} /\left(e E_{S}\right)^{2}$ in Eq (20) and to go over to the rate of created pairs. Consequently,

$$
\begin{aligned}
\dot{\mathcal{N}} \equiv & \frac{\mathcal{N}}{T} \approx \frac{\left(e E_{s}\right)^{3}}{2 \pi^{3} \omega^{2}} \int_{0}^{\pi} d \gamma_{\|} \int_{\gamma}^{\infty} d s \\
& \times s \exp \left[-\frac{e E_{s}}{\omega^{2}} s^{2} \operatorname{Im} \mathcal{S}_{0}\left(s, \gamma_{\|}\right)\right],
\end{aligned}
$$

where Eq. (14) has been inserted. Observe that the function $s^{2} \operatorname{Im} \mathcal{S}_{0}\left(s, \gamma_{\|}\right)$grows monotonically in both $s$ and $\gamma_{\|}$and $\frac{e E_{s}}{\omega^{2}} s^{2} \operatorname{Im} \mathcal{S}_{0}\left(s, \gamma_{\|}\right) \geq \frac{E_{\mathrm{cr}}}{E_{s}} \operatorname{Im} \mathcal{S}_{0}\left(\gamma, \gamma_{\|}\right) \gg 1{ }^{5} \quad$ Therefore, we integrate by parts in $s$ and expand the resulting integrand around $\gamma_{\|} \sim 0$. Consequently,

$$
\begin{aligned}
\dot{\mathcal{N}} \approx & \frac{\left(e E_{s}\right)^{2}}{8 \pi^{4}} \frac{\operatorname{erf}\left(\sqrt{\frac{1}{2} \frac{m}{\omega} h_{\varepsilon}(\gamma)} \pi\right)}{1-\mathscr{q}_{\varepsilon}(\gamma)-\frac{1}{2} \gamma \mathscr{q}_{\varepsilon}^{\prime}(\gamma)} \\
& \times \sqrt{\frac{2 \pi \omega}{m h_{\varepsilon}(\gamma)}} \exp \left[-\pi \frac{m^{2}}{e E_{s}}\left\{1-\mathscr{q}_{\varepsilon}(\gamma)\right\}\right]
\end{aligned}
$$

with $\operatorname{erf}(x)=\frac{2}{\sqrt{\pi}} \int_{0}^{x} d t e^{-t^{2}}$ denoting the error function [74]. The formula above constitutes our main analytic result.

\footnotetext{
${ }^{5} \mathrm{We}$ note that the insertion of Eq. (17) into the condition $\frac{E_{\mathrm{cr}}}{E_{s}} \operatorname{Im} \mathcal{S}_{0}\left(\gamma, \gamma_{\|}\right) \gg 1$ provides a restriction $E_{s} \gg \frac{1}{2} E_{w} e^{\frac{\omega}{4 m}}$, which is similar to the one given at the end of Sec. III.
}

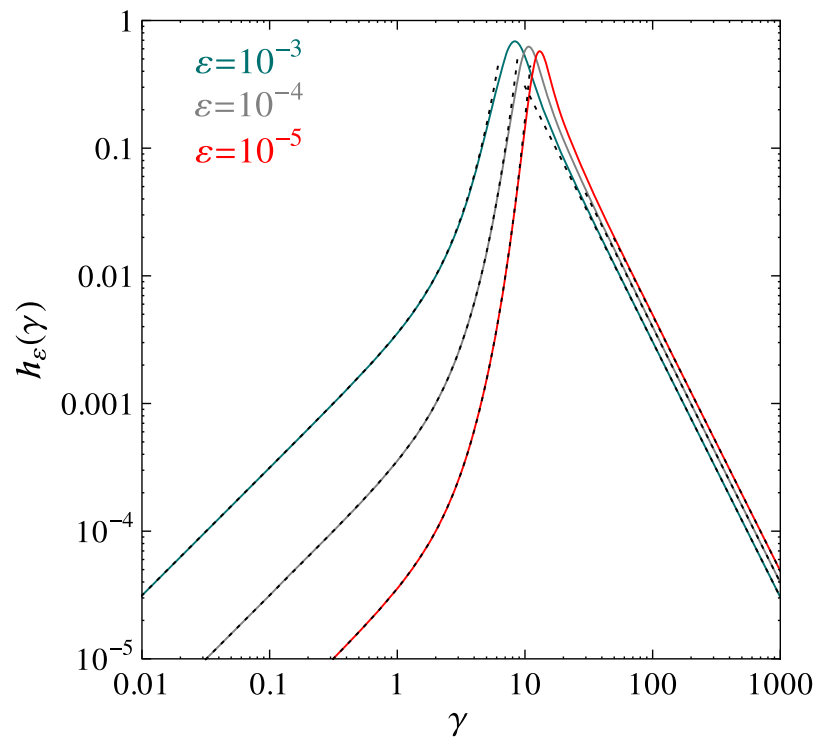

FIG. 4. Behavior of the function $h_{\varepsilon}(\gamma)$ [see Eq. (24)]. The asymptotic trends [Eq. (25)] linked to each curve are shown in dotted style.

It complements insights already gained by numerical studies [25-30] by allowing us in particular to obtain the scaling behavior of the process with the field parameters. This offers genuine advantages for optimizing the pair-production yield and discriminating it from undesirable backgrounds. Notice that its preexponential portion contains the functions $\mathscr{g}_{\varepsilon}(\gamma)$ and $\mathscr{q}_{\varepsilon}^{\prime}(\gamma) \equiv \partial \mathscr{g}_{\varepsilon}(s) /\left.\partial s\right|_{s=\gamma}$ with

$$
\begin{aligned}
\mathscr{g}_{\varepsilon}(\gamma)= & 1-\frac{1}{\pi} \operatorname{Im} \mathcal{S}_{0}(\gamma, 0) \\
& \approx\left\{\begin{array}{lll}
\frac{2 \varepsilon}{\gamma} I_{1}(\gamma) & \text { for } & \gamma \ll 1, \\
1-\frac{4}{\pi} \frac{\gamma_{\mathrm{cr}}}{\gamma} & \text { for } & \gamma \gg \max \left\{1, \gamma_{\mathrm{cr}}\right\}
\end{array}\right.
\end{aligned}
$$

characterizing the decrement of the exponential function. As before, $\mathcal{I}_{1}(x)=\frac{x}{2}+\frac{x^{3}}{2^{2} 4}+\frac{x^{5}}{2^{2} 4^{2} 6}+\cdots$ refers to the modified Bessel function of the first kind with order 1 [74]. Additionally, Eq. (22) introduces the function

$$
\begin{aligned}
h_{\varepsilon}(\gamma) \equiv & \left.\gamma \frac{\partial^{2}}{\partial \gamma_{\|}^{2}} \operatorname{Im} \mathcal{S}_{0}\left(\gamma, \gamma_{\|}\right)\right|_{\gamma_{\|}=0} \\
= & 8 \varepsilon \gamma \int_{0}^{1} d y \frac{\sqrt{1-y^{2}}}{[1+\varepsilon \cosh (\gamma y)]^{3}} \\
& \times\left[\frac{1}{2} \cosh (\gamma y)+\varepsilon\left(1-\frac{1}{2} \cosh ^{2}(\gamma y)\right)\right],
\end{aligned}
$$

the behavior as a function of the parameter $\gamma$ of which is depicted in Fig. 4. All curves exhibited there remain below $1\left[h_{\varepsilon}(\gamma)<1\right]$. They manifest fast decaying laws for both $\gamma \gg 1$ and $\gamma \ll 1$. These trends can be understood when looking for their asymptotes, which turn out to be 

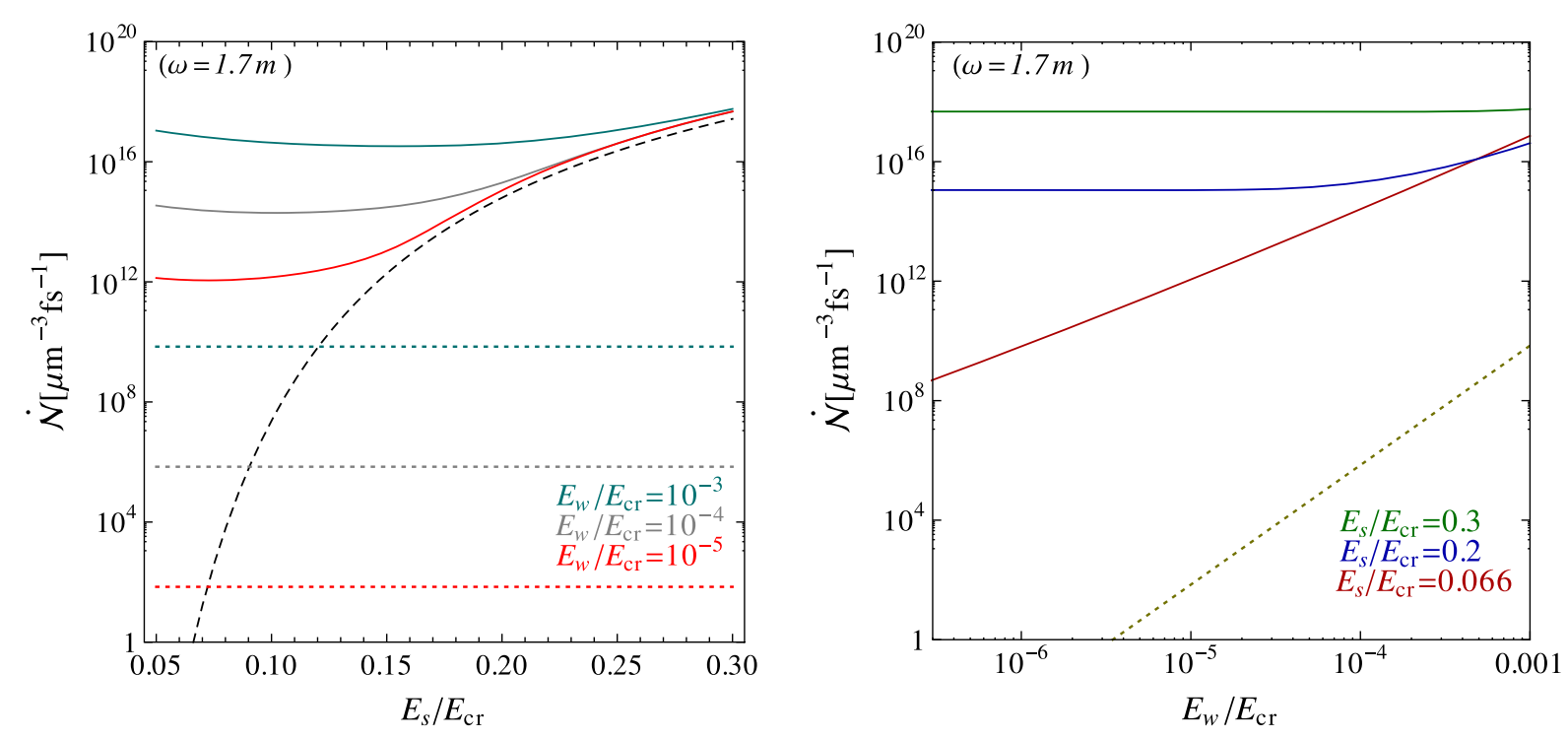

FIG. 5. Pair-production rate per unit of volume in a field configuration in which a constant strong field is assisted by a weak but fastoscillating electric mode. Its dependences on the strong and weak fields are shown in the left and right panels, respectively. In the left panel, the dashed curve results from the standard Schwinger mechanism, whereas the horizontal dotted lines have been obtained by considering the effect of the fast-oscillating field only. Likewise, in the right panel, the dotted curve in olive describes the behavior of the rate when the strong field is not present and only the perturbative mode drives the pair-creation process. The corresponding expression associated with this scenario can be found in Refs. [75-77].

$$
h_{\varepsilon}(\gamma) \approx\left\{\begin{array}{ccc}
2 \pi \varepsilon I_{1}(\gamma) & \text { for } & \gamma \ll 1, \\
4 \frac{\gamma_{\mathrm{cr}}}{\gamma^{2}} & \text { for } & \gamma \gg \max \left\{1, \gamma_{\mathrm{cr}}\right\} .
\end{array}\right.
$$

We note that the expression linked to the case $\gamma \ll 1$ can even be applied to study the behavior of $h_{\varepsilon}(\gamma)$ in regions for which $\gamma \gtrsim 1$. The dotted curves in the left portion of Fig. 4 reveal to us that the loss of accuracy in such a case is almost undiscernible.

In the regime characterized by the conditions $\gamma \ll 1$ and $m<\omega \lesssim 2 m$, the argument of the error function is very small, and its leading order contribution behaves as erf $(x) \sim$ $2 x / \sqrt{\pi}$. In such a scenario, Eq. (22) reproduces the known rate for the Schwinger mechanism (quasistatic limit)

$$
\dot{\mathcal{N}} \approx \frac{\left(e E_{s}\right)^{2}}{4 \pi^{3}} \exp \left[-\pi \frac{E_{\mathrm{cr}}}{E_{s}}\right] .
$$

When $\gamma$ exceeds unity and the critical value $\gamma_{\text {cr }}$ significantly $\left(\gamma \gg 1\right.$ and $\gamma \gg \gamma_{\text {cr }}$ ) with $m<\omega \lesssim 2 m$, the small-argument behavior of the error function still can be applied to Eq. (22), and the production rate approximates

$$
\dot{\mathcal{N}} \approx \frac{e E_{s}}{8 \pi^{2}} \frac{m \omega}{\ln \left[2 \frac{E_{s}}{E_{w}}\right]}\left[\frac{1}{2} \frac{E_{w}}{E_{s}}\right]^{\frac{4 m}{\omega}} .
$$

Interestingly, this formula manifests a nonperturbative dependence not only in $E_{s}$ but also in the field strength $E_{w}$ associated with the weak electric mode. Clearly, in the intermediate regime not covered by these asymptotic cases, the density rate for pair production [see Eq. (22)] mixes both tunneling and multiphoton effects.

In order to extend further our knowledge on $\dot{\mathcal{N}}$, we show in Fig. 5 its behavior as a function of the strong (left panel) and weak (right panel) field strengths. The results exhibited in both panels have been obtained by setting the frequency of the fast-oscillating field to $\omega=1.7 \mathrm{~m}$. Following the discussion at the end of Sec. III, the left panel has been generated by varying the strong field between $5 \times 10^{-2} \leq E_{s} / E_{\mathrm{cr}} \leq 3 \times 10^{-1}$. Here, the dashed curve corresponds to the standard Schwinger mechanism [see Eq. (26)], whereas the dotted curves result from the case in which the pair-production process is driven by the fast-oscillating mode only. The expression used to generate the curves linked to the latter scenario is given in Refs. [75-77]. Here, by increasing the weak field amplitude by an order of magnitude, the production rate grows by a factor $10^{4}$, indicating that the process occurs in the perturbative regime with absorption of two quanta $\omega$. For comparison, we note that the curves for the assisted setup differ by relative factors of about 200 each at $E_{s} \approx 6.6 \times 10^{-2} E_{\mathrm{cr}}$. We observe besides that, on average, the slope linked to the rate of the standard Schwinger effect is larger than the ones corresponding to the assisted setup. Hence, as the ratio $E_{s} / E_{\text {cr }}$ grows, the enhancing due to the fast-oscillating field becomes less pronounced. This fact corroborates the idea that, in an assisted scenario, there exist two channels for increasing the pair-production rate, either by growing the strong field strength or via the absorption of quanta from the fast-oscillating mode. The 


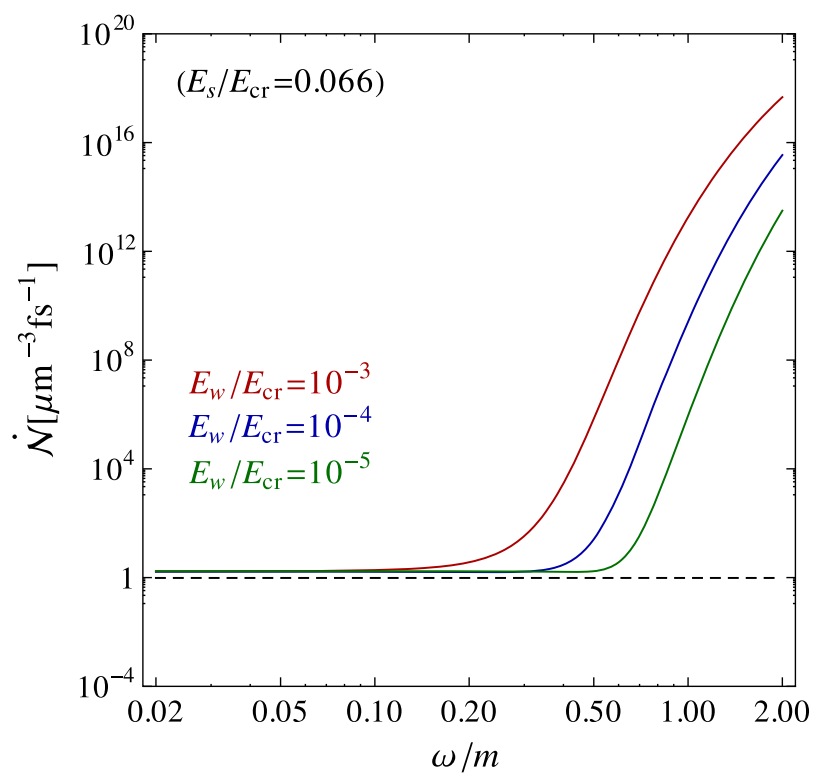

FIG. 6. Frequency dependence of the pair-production rate per unit of volume associated with the assisted Schwinger mechanism. Here, the horizontal dashed line follows from the expression associated with the Schwinger mechanism.

former path rules the process as $E_{s}$ grows, while the latter dominates as the contrary condition occurs.

Figure 6 is intended to provide insight about the trend of the rate with the change of $\omega$. It has been obtained by setting the strong field to $E_{s}=6.6 \times 10^{-2} E_{\mathrm{cr}}$. As in Fig. 5, the dashed line follows from the expression associated with the Schwinger mechanism [see Eq. (26)]. We note that all solid curves closely approach the value of the standard Schwinger mechanism when $\omega \ll m$.

We wish to put our outcomes in context. So, let us suppose an experiment driven by the strong field to be reached at the forthcoming ELI laser system. Accordingly, we take $E_{s} \approx$ $6.6 \times 10^{-2} E_{\mathrm{cr}}$ as a reference parameter. To achieve a peak field strength of this nature, a strong focusing - close to the diffraction limit - is required. Consequently, we will suppose that the spatial extent and the temporal length of the laser pulse are $\ell \sim 1 \mu \mathrm{m}$ and $\mathrm{T} \sim 1 \mathrm{fs}$, respectively. This laser is expected to operate with a central frequency $\Omega \approx 1.55 \mathrm{eV}$. Notice that, if the fast-oscillating mode operates at $\omega \approx 1.7 \mathrm{~m}$, the number of cycles it makes during the pulse length $T$ is $N \approx 2.1 \times 10^{5}$, which exceeds largely the combined Keldysh parameter $\gamma \approx 26$. Under such a circumstance, the number of pairs yielded from an assisted configuration characterized by $E_{w}=10^{-3} E_{\mathrm{cr}}$ is $n_{e^{-} e^{+}}=$ $\dot{\mathcal{N}} \ell^{3} T \approx 7.4 \times 10^{16}$ (curve in cyan in the left panel of Fig. 5), which would exceed the result associated with the Schwinger mechanism (black dashed curve) $n_{e^{-} e^{+}} \sim 1$ by 16 orders of magnitude roughly. However, when comparing this value with the dotted curve in cyan-corresponding to the case in which PP is due to the fast-oscillating mode only—we find $n_{e^{-} e^{+}} \approx 7 \times 10^{9}$, which leads to an effective enhancement by 7 orders of magnitude, approximately.
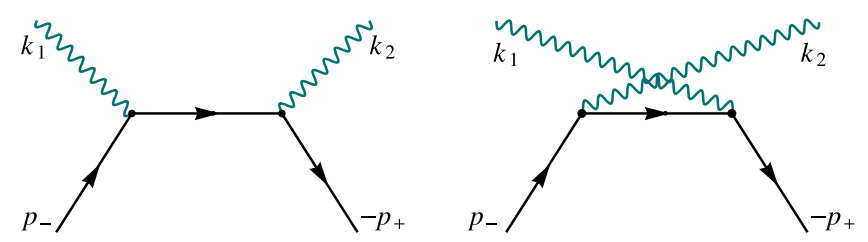

FIG. 7. Feynman diagrams contributing to the electron-positron recombination into two photons.

Some comments are in order. First, we note that the discussed improvements have been obtained from a single laser shot only. Certainly, our study provides evidences that the pair-production enhancement increases significantly for frequencies $m \lesssim \omega<2 m$. We note that, in such an energy regime, differences between pair production in an oscillating electric field and pair production in a standing laser wave have been pointed out, owing to the spatial dependence and magnetic component of the latter [78-80]. Hence, the results presented here are expected to describe only qualitatively the phenomenon taking place in realistic laser fields. Notwithstanding this, the general physical conclusions drawn regarding the dependence on the electric field strengths of both the strong and the fast-oscillating fields are expected to find their counterparts in a laser-based experiment for the assisted Schwinger mechanism.

\section{HIGH-ENERGY PHOTON EMISSION AS A PROBE OF THE DYNAMICALLY ASSISTED SCHWINGER MECHANISM}

The enhancement induced by the superposition of the fast-oscillating wave onto the strong field background might facilitate the experimental verification of the spontaneous production of pairs from the vacuum, by detecting the generated particles directly. However, the high densities of residual pairs promote their recombinations (see Fig. 7), and thus the emission of photons. Hence, the created electron-positron plasma has a pronounced unstable nature, and the number of pairs evolves according to the law $n_{e^{-} e^{+}}(t)=n_{e^{-} e^{+}}[1+\delta t / \tau]^{-1}$, where $n_{e^{-} e^{+}}$is the number of pairs at the moment when the external field is switched off ( $\delta t \equiv t-T / 2=0)$, whereas $\tau$ denotes the plasma lifetime. ${ }^{6}$ An estimate for $\tau$ will be given below. It is worth remarking that the number of photons emitted simultaneously at time $t$ is

$$
\begin{aligned}
\Delta n_{\gamma}(t) & =n_{\text {total }}(t)-n_{\gamma}(T) \\
& =n_{e^{-} e^{+}} \frac{\delta t}{\tau} \frac{1}{1+\frac{\delta t}{\tau}} .
\end{aligned}
$$

While $n_{\text {total }}(t)$ denotes the total number of photons present at the time $t, n_{\gamma}(T)$ refers to the existing background of them

\footnotetext{
${ }^{6}$ This decay law arises when assuming that the associated rate is proportional to both the number of electrons and of positrons present at time $t$, i.e., for $\dot{n}_{e^{-} e^{+}}(t)=-\frac{1}{\tau} n_{e^{-} e^{+}}^{2}(t)$.
} 
when the external field is switched off. In what follows, we suppose the latter causes only a minor effect on the singleparticle distribution function $W_{T}(\boldsymbol{p})$ [see Eqs. (14), (18), and (19)]. In contrast to previous investigations [47-51,61], we will limit ourselves to the number of photons produced after the field has been switched off, i.e., when the stability of the quantum vacuum is reestablished and the two-photon emission process becomes dominant (see Fig. 7).

Clearly, in a time interval of the order of $\tau$ or larger-in addition to the electron-positron annihilation process-also scattering events of particles and antiparticles are very likely to take place. As a consequence, the initial particle spectrum is supposed to change significantly. In contrast, for a $\delta t \ll \tau$, i.e., for early times, neither the single-particle distribution function nor the initial number of pairs is expected to change appreciably $\left[n_{e^{-} e^{+}}(t) \approx n_{e^{-} e^{+}}\right]$. Likewise, the total number of emitted photons approaches

$$
\Delta n_{\gamma}(t) \approx n_{e^{-} e^{+}} \frac{\delta t}{\tau}
$$

This formula constitutes a good approximation only when $\Delta n_{\gamma}(t) \ll n_{e^{-} e^{+}}$, in which case the consequences of both annihilation and scattering processes can be treated perturbatively. Under such circumstances, the differential number of recombination events per unit volume and unit time approaches

$$
\begin{aligned}
& d \dot{\nu}=\frac{1}{2} e^{4} d^{3} k_{1} d^{3} k_{2} d^{3} p_{+}{\mathrm{d}^{3}}^{3} p_{-} \delta_{k_{1}+k_{2}, p_{+}+p_{-}}^{4} \\
& \times W_{T}\left(\boldsymbol{p}_{+}\right) W_{T}\left(\boldsymbol{p}_{-}\right) \overline{\mathcal{M}^{2}}{ }_{e^{-} e^{+} \rightarrow \gamma \gamma}\left(k_{1}, k_{2}, p_{+}, p_{-}\right),
\end{aligned}
$$

where the $1 / 2$-factor arises since the emitted photons of the final state are indistinguishable. For the sake of simplicity, the shorthand notations $\delta_{p, q}^{4}=(2 \pi)^{4} \delta^{4}(p-q), \mathbb{d}^{3} k_{1,2}=$ $d^{3} k_{1,2} /\left[2 \omega_{k_{1,2}}(2 \pi)^{3}\right]$, and $\mathrm{d}^{3} p_{ \pm}=d^{3} p_{ \pm} /\left[2 w_{p_{ \pm}}(2 \pi)^{3}\right]$ with $p_{ \pm}=\left(w_{p_{ \pm}}, \boldsymbol{p}_{ \pm}\right)$and $k_{1,2}=\left(\omega_{k_{1,2}}, \boldsymbol{k}_{1,2}\right)$ have been used. Positive and negative subscripts identify the positron and electron momentum, respectively. Notice that $w_{p_{ \pm}}=$ $\left[p_{\perp, \pm}^{2}+p_{\|, \pm}^{2}+m^{2}\right]^{1 / 2}$ is the energy of the positron and the electron when the field has been switched off. Here, $\overline{\mathcal{M}^{2}} e^{-} e^{+} \rightarrow \gamma \gamma$ denotes the unpolarized squared invariant amplitude of the annihilation process (see Fig. 7). The precise expression of this object can be found in text books—see, for instance, Ref. [81]—and reads

$$
\begin{aligned}
\overline{\mathcal{M}^{2}} e^{-} e^{+} \rightarrow \gamma \gamma & =2\left[\frac{k_{2} p_{-}}{k_{1} p_{-}}+\frac{k_{1} p_{-}}{k_{2} p_{-}}\right. \\
& \left.+\frac{2 m^{2} k_{1} k_{2}}{\left(k_{1} p_{-}\right)\left(k_{2} p_{-}\right)}-\frac{m^{4}\left(k_{1} k_{2}\right)^{2}}{\left(k_{1} p_{-}\right)^{2}\left(k_{2} p_{-}\right)^{2}}\right] .
\end{aligned}
$$

At this point, it turns out to be convenient to take into account the identity $\int \mathrm{d}^{3} k_{i}=\int \frac{d^{4} k_{i}}{(2 \pi)^{4}} \delta\left(k_{i}^{2}\right) \Theta\left(k_{i 0}\right)$ and perform the integrations over $k_{2}$ and $\omega_{\boldsymbol{k}_{1}}$. As a consequence,

$$
\begin{aligned}
\frac{d \dot{\nu}}{d \varphi d \theta \sin \theta}= & \frac{1}{2} \alpha^{2} \int \mathrm{d}^{3} p_{+} \mathrm{d}^{3} p_{-} W_{T}\left(\boldsymbol{p}_{+}\right) W_{T}\left(\boldsymbol{p}_{-}\right) \\
& \times \frac{\left(m^{2}+p_{+} p_{-}\right) \overline{\mathcal{M}^{2}} e^{-} e^{+} \rightarrow \gamma \gamma}{\left(w_{\boldsymbol{p}_{+}}+w_{\boldsymbol{p}_{-}}-\boldsymbol{p}_{-} \cdot \boldsymbol{n}-\boldsymbol{p}_{+} \cdot \boldsymbol{n}\right)^{2}},
\end{aligned}
$$

where $\alpha=1 / 137$ is the fine-structure constant. Here, $\theta$ is the polar angle that the wave vector $\boldsymbol{n}=\boldsymbol{k}_{1} /\left|\boldsymbol{k}_{1}\right|$ forms with the polarization direction of the switched-off field. In contrast, $\varphi$ represents the azimuthal angle. In the expression above, $\overline{\mathcal{M}^{2}} e^{-} e^{+} \rightarrow \gamma \gamma$ must be understood as function depending only on $p_{ \pm}$and $k_{1}^{\mu}=\omega_{\boldsymbol{k}_{1}}(1, \boldsymbol{n})$ with

$$
\omega_{\boldsymbol{k}_{1}}=\frac{m^{2}+p_{+} p_{-}}{w_{\boldsymbol{p}_{+}}+w_{\boldsymbol{p}_{-}}-\boldsymbol{p}_{-} \cdot \boldsymbol{n}-\boldsymbol{p}_{+} \cdot \boldsymbol{n}} .
$$

The integrals over $\boldsymbol{p}_{\perp, \pm}$ can also be carried out approximately. To this end, we first develop the change of variables $\gamma_{\perp, \pm}^{2}=\left(m^{2}+\boldsymbol{p}_{\perp, \pm}^{2}\right) \omega^{2} /\left(e E_{s}\right)^{2}$ and consider the fact that the exponent associated with $W_{T}\left(\boldsymbol{p}_{+}\right)\left[W_{T}\left(\boldsymbol{p}_{-}\right)\right]$grows monotonically with $\gamma_{\perp,+}\left[\gamma_{\perp,-}\right]$. Hence, after integrating over these variables by parts separately, we end up with

$$
\begin{aligned}
\frac{d \dot{\nu}}{d \varphi d \theta \sin \theta} \approx & \frac{\alpha^{2}}{32 \pi^{4}}\left(\frac{E_{s}}{E_{\mathrm{cr}}}\right)^{2} m^{4} \int_{-\frac{\pi N}{\gamma} m}^{\frac{\pi N}{\gamma} m} \frac{d p_{\|,+}}{2 w_{p_{\|,+}}} \\
& \times \int_{-\frac{\pi N}{\gamma} m}^{\frac{\pi N}{\gamma} m} \frac{d p_{\|,-}}{2 w_{p_{\|,-}}} \overline{\mathcal{M}^{2}} e_{e^{-} e^{+} \rightarrow \gamma \gamma}\left(p_{\|,+}, p_{\|,-}, \theta\right) \\
& \times \frac{m^{2}+w_{p_{\|+}} w_{p_{\|,-}}+p_{\|-} p_{\|+}}{\left[w_{p_{\|,+}}+w_{p_{\|,-}}-\left(p_{\|,+}-p_{\|,-}\right) \cos \theta\right]^{2}} \\
& \times \frac{\exp \left[-\frac{E_{\mathrm{cr}}}{E_{s}} \operatorname{Im} \mathcal{S}_{0}\left(\gamma, p_{\|,+}\right)\right]}{\left[\operatorname{Im} \mathcal{S}_{0}\left(\gamma, p_{\|,+}\right)+\frac{1}{2} \gamma \frac{\partial}{\partial \gamma} \operatorname{Im} \mathcal{S}_{0}\left(\gamma, p_{\|,+}\right)\right]} \\
& \times \frac{\exp \left[-\frac{E_{\mathrm{cr}}}{E_{s}} \operatorname{Im} \mathcal{S}_{0}\left(\gamma, p_{\|,-}\right)\right]}{\left[\operatorname{Im} \mathcal{S}_{0}\left(\gamma, p_{\|,-}\right)+\frac{1}{2} \gamma \frac{\partial}{\partial \gamma} \operatorname{Im} \mathcal{S}_{0}\left(\gamma, p_{\|,-}\right)\right]} .
\end{aligned}
$$

The applied procedure manifests that the main contribution of these integrations results from the region in which $p_{\perp, \pm} / m \ll 1$. This result is somewhat expected since the single-particle distribution function [see Eqs. (14), (18), and (19)] is sharply peaked at $p_{\perp}=0$, and the typical value of momentum perpendicular to the field direction $\left(\left\langle\boldsymbol{p}_{\perp}\right\rangle=0\right)^{7}$

\footnotetext{
${ }^{7}$ Here, $\langle\mathcal{O}\rangle=\int d^{3} p \mathcal{O}(\boldsymbol{p}) W_{\infty}(\boldsymbol{p}) / \int d^{3} p W_{\infty}(\boldsymbol{p})$. For computing the integration with $\mathcal{O}(\boldsymbol{p})=p_{\perp}^{2}$, a procedure similar to the one used for obtaining Eq. (22) has been followed.
} 


$$
\begin{aligned}
p_{\perp} & \sim\left\langle p_{\perp}^{2}\right\rangle^{1 / 2} \\
& \approx \frac{m}{\sqrt{\pi}}\left[\frac{E_{s}}{E_{\mathrm{cr}}}\right]^{1 / 2} \frac{1}{\left[1-\mathscr{q}_{\varepsilon}(\gamma)-\frac{1}{2} \gamma \mathscr{q}_{\varepsilon}^{\prime}(\gamma)\right]^{1 / 2}}
\end{aligned}
$$

remains much smaller than $m$. Hence, the photons are emitted quasi-isotropically on the polar plane, while their azimutal distribution follows a nontrivial law to be determined in brief. Still, the main trend of this angular distribution can be anticipated when noting that the typical value of momentum parallel to $\boldsymbol{E}_{s}$ grows linearly with the pulse length $\left(\left\langle\boldsymbol{p}_{\|}\right\rangle=0\right)$ :

$$
p_{\|} \sim\left\langle p_{\|}^{2}\right\rangle^{1 / 2} \approx \frac{1}{2 \sqrt{3}} e E_{s} T .
$$

As this largely exceeds $p_{\perp}$ from Eq. (35), we find that, on average, the angle between the momentum of the created particles and the external field $\theta \sim\left\langle p_{\perp}^{2}\right\rangle^{1 / 2} /\left\langle p_{\|}^{2}\right\rangle^{1 / 2} \ll 1$. Moreover, Eq. (36) provides evidence that the yielded electrons and positrons are mostly ultrarelativistic because $e E_{s} T / m=2 \pi N / \gamma \gg 1$. Indeed, their average energy turns out to be

$$
\left\langle w_{p_{ \pm}}\right\rangle \approx \frac{1}{4} e E_{s} T
$$

with a standard deviation $\Delta w_{\boldsymbol{p}_{ \pm}} \approx \pm\left\langle w_{\boldsymbol{p}_{ \pm}}\right\rangle / \sqrt{3}$. When the energy momentum balance linked to the recombination process $p_{+}^{\mu}+p_{-}^{\mu}=k_{1}^{\mu}+k_{2}^{\mu}$ is considered, it turns out thaton average- the outcoming photons are emitted back to back with a mean energy $\left\langle\omega_{\boldsymbol{k}_{1}}\right\rangle \approx\left\langle\omega_{\boldsymbol{k}_{2}}\right\rangle \approx\left\langle\omega_{p_{ \pm}}\right\rangle$. With a strong field strength $E_{s} \approx 6.6 \times 10^{-2} E_{\mathrm{cr}}$ and a pulse length $\mathrm{T} \sim 1$ fs corresponding to $N \approx 2.1 \times 10^{5}$ for $\omega=1.7 \mathrm{~m}$ $(\gamma \approx 26)$, the mean photon energy is $\left\langle\omega_{k_{1}}\right\rangle \approx 6.3 \mathrm{GeV}$.

Returning back to Eq. (34), the integrals which remain there cannot be computed analytically. To approximate them, we first develop the change of variables $\gamma_{\|, \pm}=$ $p_{\|, \pm} \omega /\left(e E_{s}\right)$ and decompose them in sums over integrals defined over regions with $2 \pi$-extensions,

$$
\begin{aligned}
\int_{-\frac{\pi N}{r} m}^{\frac{\pi N}{r} m} d p_{\|, \pm} \cdots & =\frac{e E_{s}}{\omega} \int_{-\pi N}^{\pi N} d \gamma_{\|, \pm} \cdots \\
& =\frac{e E_{s}}{\omega} \sum_{m} \int_{(2 m-1) \pi}^{(2 m+1) \pi} d \gamma_{\|, \pm} \cdots
\end{aligned}
$$

where $m$ runs from $m_{\min }=-\lfloor(N-1) / 2\rfloor$ to $m_{\max }=$ $\lfloor(N-1) / 2\rfloor$ with $\lfloor x\rfloor$ referring to the integer value of $x$. Observe that the main contribution of each individual integration results from the region of $\gamma_{\|, i} \in[(2 m-1) \pi$, $(2 m+1) \pi]$ for which the exponent is minimized. Following our discussion in Sec. III, this takes place at $\gamma_{\|, i}=2 m \pi$. Hence, we expand each exponent up to the order $\left(\gamma_{\|, i}-\right.$ $2 m \pi)^{2}$ and set $\gamma_{\|, i}=2 m \pi$ in each preexponent. Afterward, $\gamma_{\|, \pm}$are integrated out, and we obtain

$$
\frac{d \dot{\nu}}{d \varphi d \theta \sin \theta} \approx \frac{\alpha^{2} \pi^{2}}{(m \omega)^{2}} \dot{\mathcal{N}}^{2} \mathfrak{F}(\gamma, \theta),
$$

where Eq. (22) has been inserted. Here, the function $\mathfrak{F}(\gamma, \theta)$ encodes the angular distribution in the polar plane and reads

$$
\begin{aligned}
\mathfrak{F}(\gamma, \theta)= & \sum_{m, m^{\prime}} \frac{Q_{m, m^{\prime}}}{\mathcal{E}_{m} \mathcal{E}_{m^{\prime}}\left[f_{m}(\theta)+f_{m^{\prime}}(\theta)\right]^{2}} \\
& \times\left[\frac{f_{m}(\theta)}{f_{m^{\prime}}(\theta)}+\frac{f_{m^{\prime}}(\theta)}{f_{m}(\theta)}+\frac{2\left[f_{m}(\theta)+f_{m^{\prime}}(\theta)\right]^{2}}{f_{m}(\theta) f_{m^{\prime}}(\theta) Q_{m, m^{\prime}}}\right. \\
& \left.-\frac{\left[f_{m}(\theta)+f_{m^{\prime}}(\theta)\right]^{4}}{f_{m}(\theta)^{2} f_{m^{\prime}}(\theta)^{2} Q_{m, m^{\prime}}^{2}}\right] .
\end{aligned}
$$

Other functions contained in the expression above are given by

$$
\begin{aligned}
Q_{m, m^{\prime}} & =1+\frac{2 m \pi}{\gamma} \frac{2 m^{\prime} \pi}{\gamma}+\mathcal{E}_{m} \mathcal{E}_{m^{\prime}}, \\
f_{m}(\theta) & =\mathcal{E}_{m}-\frac{2 m \pi}{\gamma} \cos \theta, \\
f_{m^{\prime}}(\theta) & =\mathcal{E}_{m^{\prime}}+\frac{2 m^{\prime} \pi}{\gamma} \cos \theta, \\
\mathcal{E}_{m} & =\sqrt{1+\frac{4 \pi^{2} m^{2}}{\gamma^{2}}}, \quad \mathcal{E}_{m^{\prime}}=\sqrt{1+\frac{4 \pi^{2} m^{\prime 2}}{\gamma^{2}}} .
\end{aligned}
$$

An estimate for the number of emitted photons $\Delta n_{\gamma}(t)=\Delta \nu \ell^{3}$ in a volume $\ell^{3}$ can be established from Eq. (39) and reads

$$
\Delta n_{\gamma}(t) \approx \frac{4 \alpha^{2} \pi^{3}}{(m \omega)^{2}} \dot{\mathcal{N}} n_{e^{-} e^{+}} \frac{\delta t}{T} \int_{0}^{1} d x \mathfrak{F}(\gamma, x),
$$

where the change of variable $x=\cos \theta$ has been carried out and

$$
\begin{aligned}
\int_{0}^{1} d x \mathfrak{F}(\gamma, x)= & 1-\sum_{m, m^{\prime} \neq 0} \frac{Q_{m, m^{\prime}}}{\mathcal{E}_{m} \mathcal{E}_{m^{\prime}}}\left[\frac{2 \mathcal{G}_{m, m^{\prime}}}{\mathcal{E}_{m}+\mathcal{E}_{m^{\prime}}}\right. \\
& -\left(1+\frac{2}{Q_{m, m^{\prime}}}+\frac{2}{Q_{m, m^{\prime}}^{2}}\right) \frac{\ln \left[\frac{\mathcal{E}_{m}}{\mathcal{E}_{m^{\prime}}} \frac{f_{m_{m}^{\prime}}(1)}{f_{m}(1)}\right]}{\frac{2 \pi m^{\prime}}{\gamma} \mathcal{E}_{m}+\frac{2 \pi m}{\gamma} \mathcal{E}_{m^{\prime}}} \\
& \left.+\frac{1}{Q_{m, m^{\prime}}^{2}}\left(\frac{1}{\mathcal{E}_{m^{\prime}} f_{m^{\prime}}(1)}+\frac{1}{\mathcal{E}_{m} f_{m}(1)}\right)\right]
\end{aligned}
$$

Here, the function $\mathcal{G}_{m, m^{\prime}}$ reads

$$
\mathcal{G}_{m, m^{\prime}}=\left\{\begin{array}{lll}
1 & \text { for } & m=m^{\prime}, \\
\frac{1}{f_{m}(1)+f_{m^{\prime}}(1)} & \text { for } & m \neq m^{\prime} .
\end{array}\right.
$$

It is worth remarking that, to be consistent with our earlytime requirement [see below Eq. (29)], the relation $\Delta n_{\gamma}(t) \ll n_{e^{-} e^{+}}$has to be satisfied. Observe that such a 


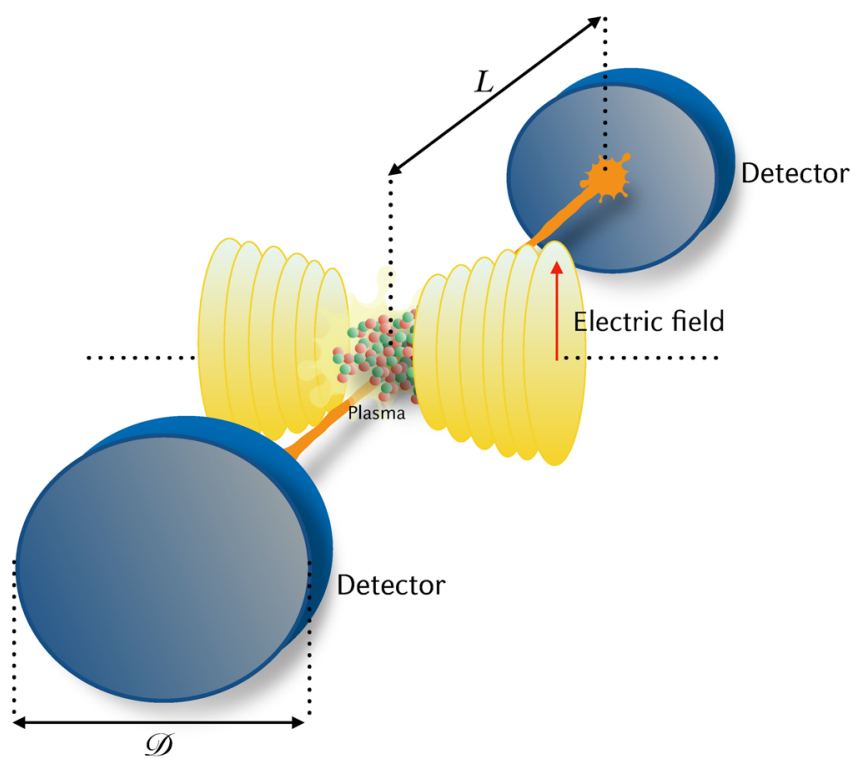

FIG. 8. Schematic diagram for an experimental setup which probes the possible realization of the dynamical assisted Schwinger mechanism via the emission of high-energy photons (in orange) coming out from electron-positron annihilations of the residual plasma.

condition translates into $\delta t \ll \tau$, where the characteristic timescale ruling the perturbative treatment is given by

$$
\tau=T\left[\frac{4 \alpha^{2} \pi^{3}}{(m \omega)^{2}} \dot{\mathcal{N}} \int_{0}^{1} d x \mathfrak{F}(\gamma, x)\right]^{-1} .
$$

This expression can be interpreted as a relaxation time of an electron (positron) till annihilation occurs. We point out that analogous expressions are obtained when elastic scattering processes are considered instead. However, as the differential cross sections linked to such phenomena are comparable in magnitude with the one associated with the annihilation process, the corresponding timescales should not differ significantly from the one given above. Observe that the $\dot{\mathcal{N}}^{-1}$ dependence exhibited in Eq. (45) indicates explicitly that this timescale becomes shorter as the density of pairs increases. Consequently, the enhancement supplied by the assisted setup might not favor the direct detection of the yielded electron-positron pairs. To have a notion about plausible values of $\tau$, we refer to the results shown in the left panel of Fig. 5. For a field configuration characterized by $E_{s} \approx 6.6 \times 10^{-2} E_{\mathrm{cr}}, E_{w}=10^{-3} E_{\mathrm{cr}}$, a pulse length $\mathrm{T} \sim 1 \mathrm{fs}-$ corresponding to $N \approx 2.1 \times 10^{5}$ for $\omega=1.7 m$-the combined Keldysh parameter is $\gamma \approx 26, \int_{0}^{1} d x \mathfrak{F}(\gamma, x) \approx 5.5 \times$ $10^{4}$ and $\dot{\mathcal{N}} \approx 7.4 \times 10^{16} \mathrm{fs}^{-1} \mu \mathrm{m}^{-3}$. Under such a circumstance, the relaxation time amounts to $\tau \approx 1.3 \mathrm{~ns}$. This number exceeds - to establish a comparison-the parapositronium lifetime ( $\tau_{0} \approx 0.12 \mathrm{~ns}$ ) by an order of magnitude.

Alternative detection routes allowing us to exploit the enhancement induced by the assisted Schwinger mechanism are desirable. Motivated by this demand, we put forward a plausible experimental setup for verifying the dynamically assisted Schwinger mechanism via the detection of photons resulting from the recombination of yielded electronpositron pairs. We propose a scenario in which the highdensity electron-positron plasma is generated between two photon detectors, both placed perpendicular to the polarization direction of the strong field $(\theta=\pi / 2)$. We suppose both of them equidistant from the plasma region at a distance $L \approx 2 \times 10^{2} \mathrm{~cm}$ with their centers forming a right angle with respect to the propagation direction of the colliding pulses (see Fig. 8). In addition, we will assume both detectors characterized by a length scale $\mathcal{D} \approx 1 \mathrm{~cm}$, so that the angular openings on the polar and azimuthal planes are very small $\left(\delta \varphi, \delta \theta \approx 10^{-2} \mathrm{rad}\right.$ ). With all these details in mind, we can proceed-starting from Eq. (39) and taking into account Eq. (45) - to estimate the amount of photons reaching a detector due to the recombination after the field has been switched off,

$$
n_{\mathrm{det}} \sim n_{e^{-} e^{+}} \frac{\beta \delta \varphi \delta \theta \mathfrak{F}(\gamma, x=0)}{4 \pi \int_{0}^{1} d x \mathfrak{F}(\gamma, x)},
$$

where the parameter $\beta=\delta t / \tau$ accounts for the smallness of $\delta t$ relative to $\tau$ [see Eq. (45)]. In order to satisfy safely the early-time restriction, we take $\beta=10^{-2}$; i.e., the measurement time should be $\delta t \approx 10 \mathrm{ps}$. It is worth remarking that for our reference parameters - see the paragraph that follows Eq. (45)- the form factor attains the value $\mathfrak{F}(\gamma, x=0) \approx$ $6.9 \times 10^{3}$. With these details in mind, we find that $n_{\mathrm{det}} \approx$ $7.4 \times 10^{8}$ photons should be detected. Notably, if the weak field is reduced by 2 orders of magnitude while the remaining parameters keep their values, the initial number of pairs changes to $n_{e^{-} e^{+}} \approx 1.2 \times 10^{12}$ (see red curve in Fig. 5), and the number of photons to be detected $n_{\text {det }} \approx$ $1.2 \times 10^{4}$ appears still viable experimentally.

\section{CONCLUSIONS}

An analytical investigation of the assisted Schwinger mechanism has been carried out starting from the lowdensity approximation to the single-particle distribution function. We have revealed fundamental aspects associated with this process when a weak oscillating field mode is superimposed onto a strong constant field background. It has been shown that the particle spectrum is characterized by tiny oscillations along the external field direction, whereas perpendicular to it, the spectrum falls with the growing of $p_{\perp}$ significantly more slowly than in the case where the production process is driven by the strong field only. Once the field has been switched off, the meansquared values of the momentum depend nontrivially on the external field parameters, and the quantity linked to the parallel component to the field exceeds largely the one associated with the perpendicular momentum. As a 
consequence, most of the particles yielded at that time move approximately parallel to the field direction. Likewise, we have found that the created plasma is composed mostly of ultrarelativistic particles and antiparticles.

Both the single-particle distribution function and the density rate of yielded pairs depend on the strong and weak field strengths in a nonperturbative way. While the pair production is predicted to increase significantly in a dynamically assisted setup, the yielded electron-positron plasma has a pronounced unstable nature. This feature demanding to carry out experimental measurement in time intervals significantly smaller than the plasma lifetime; otherwise, the beneficial aspect conceded by the assisted setup is lost. In connection, we have argued that - under the early-time circumstance- the number of photons emitted as a result of electron-positron recombinations could be large enough to constitute an indirect signal of the spontaneous production of pairs from the vacuum. Based on this effect, a plausible experimental setup for their observation has been put forward. The robustness of our estimates for the number of photons to be detected supports the viability of the proposed setup as a genuine channel for verifying the Schwinger mechanism, provided strong field strengths comparable to those to be reached at ELI and XCELS lasers are exploited.

\section{ACKNOWLEDGMENTS}

This work has been funded by the Deutsche Forschungsgemeinschaft (DFG, German Research Foundation) under Grant No. 388720772 (MU 3149/5-1).

\section{APPENDIX A: SUM OF INTEGRALS OVER $c_{k}$}

This Appendix is devoted to determining the contribution due to the poles circumvented by the chosen integration path (see Fig. 1). Explicitly,

$$
\sum_{\hbar} \oint_{\ell_{\hbar}} \frac{d \tau}{1+\tau^{2}} \exp \left[\frac{2 i \epsilon_{\perp}^{2}}{e E_{s}} \int_{0}^{\tau} d \tilde{\tau} f(\tilde{\tau})\right],
$$

where the function involved in the exponent is

$$
f(\tilde{\tau})=\frac{\left(1+\tilde{\tau}^{2}\right)^{1 / 2}}{1+\varepsilon \cos \left(\gamma_{\perp} \tilde{\tau}-\gamma_{\|}\right)} .
$$

Over each circle $c_{\iota_{\iota}}$, we have $\tau=\tau_{+\varkappa}+r / \gamma_{\perp} e^{i \varphi}$ with $3 \pi / 2 \leq \varphi<-\pi / 2$, and $r$ is an infinitesimal quantity $(r \rightarrow 0)$. Consequently,

$$
\begin{aligned}
\sum_{\hbar} \oint_{c_{k}} \ldots \approx & \frac{i \gamma}{\gamma_{\perp}} \sum_{\hbar} \frac{1}{1+\tau_{+\hbar}^{2}} \int_{3 \pi / 2}^{-\pi / 2} d \varphi \\
& \times \exp \left[i \varphi+\frac{2 i \epsilon_{\perp}^{2}}{e E_{s}} \int_{0}^{\tau_{+\hbar}+\frac{r}{\gamma_{\perp}} e^{i \varphi}} d \tilde{\tau} f(\tilde{\tau})\right] .
\end{aligned}
$$

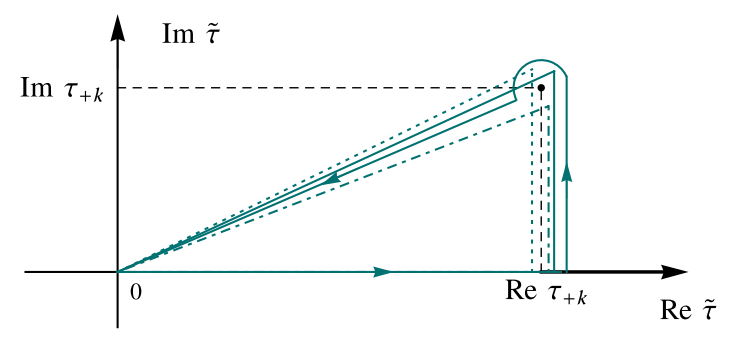

FIG. 9. Four plausible integration contours chosen to estimate the behavior of the integral involved in the exponent of Eq. (A3) as a function of $r$. Here, we have assumed $\operatorname{Re} \tau_{+k}>0$. The dotted path applies when the angle $\varphi$ lies in the sector $(\pi / 2,3 \pi / 2]$, whereas the trajectory in dot-dashed style is suitable when $-\pi / 2<\varphi<\arg \tau_{+k}$. None of these contours encloses the pole at $\tilde{\tau}=\tau_{+\kappa}$. However, the solid curve in which the arc is not present shows a plausible integration path applicable when $\arg \tau_{+\nprec}<\varphi<\pi / 2$. Conversely, the trajectory including the arc will apply if $\varphi=\arg \tau_{+\kappa}$. The corresponding integration circuits linked to the case $\operatorname{Re} \tau_{+\hbar}<0$ result from reflections with respect to the imaginary axis $(\operatorname{Re} \tilde{\tau} \rightarrow-\operatorname{Re} \tilde{\tau})$ and by taking a counterclockwise sense.

In the following, we will show that the dependence on $r$ of the integral over $\varphi$ guarantees that, at $r \rightarrow 0$, the right-hand side of Eq. (A3) vanishes. To this end, we will focus on determining the leading-order dependence of the integral involved in the exponent. While our exposition will center on the sector in which $\operatorname{Re} \tau_{+\kappa}>0$, an extension to the remaining case, i.e., $\operatorname{Re} \tau_{+\ell}<0$, is straightforward.

The first step toward our aim is to deform appropriately the integration contour of the integral involved in the exponent [see Eq. (A3)]. However, this procedure depends on the value of $\varphi$. Indeed, if $\pi / 2<\varphi \leq 3 \pi / 2$ or $-\pi / 2<\varphi<\arg \tau_{+\kappa}$, the path can be chosen without enclosing the pole $\tau_{+\kappa}$. Two plausible circuits, covering the described situation, are shown Fig. 9 in dotted and dot-dashed styles. Conversely, if $\arg \tau_{+\kappa} \leq \varphi \leq \pi / 2$, the contour is chosen such that the pole is enclosed by the trajectory. Observe that those cases in which $\varphi=\arg \tau_{+\kappa}$ or $\varphi=\pi / 2$ demand surrounding the pole with a small arc of radius $r / \gamma_{\perp}$ (see Fig. 9). Obviously, the situation described previously implies that the integral over $\varphi$ has to be split into three portions, each of which covers one of the described $\varphi$-sectors:

$$
\begin{aligned}
\int_{3 \pi / 2}^{-\pi / 2} d \varphi \ldots= & \lim _{\eta_{1}, \eta_{2} \rightarrow 0^{+}}\left[\int_{3 \pi / 2}^{\pi / 2+\eta_{1}} d \varphi \ldots\right. \\
& \left.+\int_{\pi / 2-\eta_{1}}^{\arg \tau_{+\hbar}+\eta_{2}} d \varphi \ldots+\int_{\arg \tau_{+\hbar-}-\eta_{2}}^{-\pi / 2} d \varphi \ldots\right] .
\end{aligned}
$$

Let us consider the integration over the path enclosing the pole via the arc (see Fig. 9). As a consequence of the residue theorem $\left(\operatorname{Re} \tau_{+\nprec}>0\right)$, 


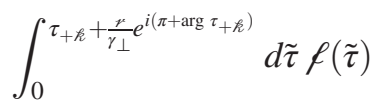

$$
\begin{aligned}
& =2 \pi i \operatorname{Res}\left[\tau_{+\nprec}, f(\tau)\right]+\mathcal{J}_{\operatorname{arc}}(\mu) \\
& +\mathcal{J}_{\operatorname{Re} \tau}(r)+\mathcal{J}_{\text {ver }}(r), \\
& \mathcal{J}_{\operatorname{arc}}(\mu)=\frac{i \mu}{\gamma_{\perp}} \int_{\arg \tau_{+\kappa}}^{\pi+\arg \tau_{+\hbar}} d \phi e^{i \phi} f\left(\tau_{+\kappa}+\frac{\mu}{\gamma_{\perp}} e^{i \phi}\right), \\
& \mathcal{J}_{\operatorname{Re} \tau}(\mu)=\int_{0}^{\operatorname{Re} \tau_{+\kappa}+\frac{\mu}{\gamma_{\perp}} \cos \left(\arg \tau_{+\hbar k}\right)} d x f(x), \\
& \mathcal{J}_{\text {ver }}(\not)=i \int_{0}^{\operatorname{Im} \tau_{+\hbar}+\frac{\varkappa}{\gamma_{\perp}} \sin \left(\arg \tau_{+\kappa}\right)} d y \\
& \times f\left(\operatorname{Re} \tau_{+\kappa}+\frac{\gamma}{\gamma_{\perp}} \cos \left(\arg \tau_{+\kappa}\right)+i y\right) .
\end{aligned}
$$

In those cases in which the integration contour avoids the pole, the Cauchy theorem applies, and the integral of interest, i.e., the left-hand side in the first line of Eq. (A5) with $\pi+\arg \tau_{+\kappa} \rightarrow \varphi$, is determined by two contributions similar to $\mathcal{J}_{\operatorname{Re} \tau}(\gamma)$ and $\mathcal{J}_{\text {ver }}(\gamma)$, with $\arg \tau_{+\varkappa} \rightarrow \varphi$ and $\varphi$ taking values within the respective sector [see below Eq. (A3)]. Conversely, if the inclusion of the pole is required without the necessity of a circumventing arc, the expression for the integral involved in the exponent will coincide with Eq. (A5) up to $\mathcal{J}_{\text {arc }}(r)$, provided the replacement $\pi+$ $\arg \tau_{+\varkappa} \rightarrow \varphi$ in the first line of Eq. (A5) is carried out. Likewise, one will be forced to replace arg $\tau_{+\varkappa} \rightarrow \varphi$ in both $\mathcal{J}_{\operatorname{Re} \tau}(r)$ and $\mathcal{J}_{\text {ver }}(r)$. Thus, the analysis of the expression above allows us to infer the outcomes related to the diverse $\varphi$ values.

Both the residue of the function $f(\tilde{\tau})$ at $\tau_{+\nprec}$ as well as the leading-order contribution linked to the tiny arc in Fig. 9,

$$
\mathcal{J}_{\text {arc }}(r) \stackrel{\kappa \rightarrow 0}{\approx}-\frac{\pi}{\gamma_{\perp}}\left(1+\tau_{+\varkappa}^{2}\right)^{1 / 2},
$$

are independent of $r$. Regarding the behavior of the integral $\mathcal{J}_{\operatorname{Re} \tau}(r)$, i.e., the third contribution in the right-hand side of Eq. (A5), here the oscillatory contribution present in its integrand is always much smaller than unity. As a consequence, one can ignore its effect by approaching $\left[1+\varepsilon \cos \left(\gamma_{\perp} x-\gamma_{\|}\right)\right]^{-1} \approx 1$, and the integral

$\lim _{\gamma \rightarrow 0} \mathcal{J}_{\operatorname{Re} \tau}(r) \approx \frac{1}{2}\left[\operatorname{Re} \tau_{+\varkappa} \sqrt{1+\left[\operatorname{Re} \tau_{+\ell}\right]^{2}}+\sin ^{-1}\left(\operatorname{Re} \tau_{+\varkappa}\right)\right]$

with $\sqrt{1+\left[\operatorname{Re} \tau_{+\varkappa}\right]^{2}} \geq 0$ becomes independent of $r$ as $\mu \rightarrow 0$.

Now, we focus on the last integral $\mathcal{J}_{\text {ver }}(r)$ in Eq. (A5), the calculation of which requires a somewhat elaborate procedure, mainly because its dependence on $\operatorname{Im} \tau_{+\kappa}$ leads to analyze the regimes $\operatorname{Im} \tau_{+\kappa} \ll 1$ and $\operatorname{Im} \tau_{+\kappa} \gg 1$ separately. As this is the only plausible contribution which may provide a nontrivial dependence on $r$, we will replace in it $\arg \tau_{+\kappa}$ by $\varphi$ and consider $\varphi$ taking values within the integration region in Eq. (A3).

\section{Case of strong enhancement: $\operatorname{Im} \tau_{+k} \ll 1$}

Let us consider first the situation in which $\operatorname{Im} \tau_{+\ell} \ll 1$. ${ }^{8}$ Because of this, the square root involved in the integrand of $\mathcal{J}_{\text {ver }}(r)$ is approximately independent of $y \equiv \operatorname{Im} \tilde{\tau}$ : $\left(1+\left[\operatorname{Re} \tau_{+\kappa}+i y\right]^{2}\right)^{1 / 2} \approx \sqrt{1+\left[\operatorname{Re} \tau_{+\kappa}\right]^{2}}$. Indeed, when the condition $\operatorname{Im} \tau_{+\kappa} \ll 1$ holds, one can exploit the fact that $y$ is much smaller than unity $(1 \gg y)$. Observe that this also implies that $\arg \left(1+\left[\operatorname{Re} \tau_{+\hbar}+i y\right]^{2}\right) \approx 0$, for all allowed values of $\operatorname{Re} \tau_{+\kappa}$. Consequently,

$$
\begin{aligned}
\mathcal{J}_{\text {ver }}(\varkappa) \approx & i \sqrt{1+\left[\operatorname{Re} \tau_{+\kappa}\right]^{2}} \int_{0}^{\operatorname{Im} \tau_{+\kappa}+\frac{r}{\gamma_{\perp}} \sin \varphi} d y \\
& \times \frac{1}{1-\varepsilon \cosh \left(\gamma_{\perp} y-i \gamma^{\prime} \cos \varphi\right)} .
\end{aligned}
$$

At this point, it turns out to be very convenient to perform the change of variable $s=\exp \left[\gamma_{\perp} y\right]$. The calculation of the resulting integral is simplified once its integrand is decomposed into partial fractions. The described procedure leads us to write

$$
\int_{0}^{\operatorname{Im} \tau_{+\kappa}+\frac{r}{\gamma_{\perp}} \sin \varphi} d y \ldots \stackrel{r \rightarrow 0}{\sim}-\frac{1}{\gamma_{\perp}}(\ln \gamma+i \varphi),
$$

where an unessential imaginary term independent of $\gamma$ and $\varphi$ has been omitted. Inserting Eq. (A9) into Eq. (A8), we find that the essential contribution of the integral involved in the exponent is

$$
\int_{0}^{\tau_{+\hbar}+\frac{r_{\perp}}{\gamma_{\perp}} e^{i \varphi}} d \tilde{\tau} f(\tilde{\tau})^{{ }^{2} \rightarrow 0} \sim \frac{i}{\gamma_{\perp}} \sqrt{1+\left[\operatorname{Re} \tau_{+\ell}\right]^{2}}(\ln \mu+i \varphi) .
$$

Correspondingly, the outer integration in Eq. (A3) [see also Eq. (A4)] behaves as

$$
\int_{3 \pi / 2}^{-\pi / 2} d \varphi \ldots \stackrel{\mu \rightarrow 0}{\sim} \mu^{\frac{2 \epsilon_{\perp}}{\omega}} \sqrt{1+\left[\operatorname{Re} \tau_{+\hbar}\right]^{2}},
$$

which guarantees that -in the regime particularized by the strong enhancement $\left(\operatorname{Im} \tau_{+\nprec} \ll 1\right)$-the sum over the circles eluding the poles gives no contribution to the singleparticle distribution function $W_{T}(\boldsymbol{p})$, provided the appropriate limit $\mu \rightarrow 0$ is taken.

\section{Case of weak enhancement: $\operatorname{Im} \tau_{+\ell} \gg 1$}

The pole always lies below the line $\mathcal{C}_{\mathcal{R}}$ and to the left of $\mathcal{C}_{+}$[read also the discussion above Eq. (12)]. This provides

\footnotetext{
${ }^{8}$ Note that $\operatorname{Im} \tau_{+\kappa}=\gamma_{\mathrm{cr}} / \gamma_{\perp}$ is actually independent of $k$.
} 
the following condition for the real and imaginary parts of the pole $\tau_{+\kappa}$ :

$$
\begin{aligned}
\sqrt{3}\left[\operatorname{Im} \tau_{+\ell}-1\right] & <\operatorname{Re} \tau_{+\ell}<\frac{\pi N}{\gamma_{\perp}}, \\
\operatorname{Im} \tau_{+\ell} & <\frac{1}{\sqrt{3}} \operatorname{Re} \tau_{+\nprec}+1<\frac{1}{\sqrt{3}} \frac{\pi N}{\gamma_{\perp}} .
\end{aligned}
$$

However, here, we will restrict ourselves to the case in which $\operatorname{Im} \tau_{+\kappa} \gg 1$. As a consequence, $\operatorname{Re} \tau_{+\kappa} \gg 1$, and the square root involved in the integrand of $\mathcal{J}_{\text {ver }}(r)$ [see Eq. (A5)] behaves as $\left(1+\left[\operatorname{Re} \tau_{+\varkappa}+i y\right]^{2}\right)^{1 / 2} \approx \operatorname{Re} \tau_{+\varkappa}+i y$ and

$\mathcal{J}_{\text {ver }}(r) \approx i \int_{0}^{\operatorname{Im} \tau_{+\kappa}+\frac{\gamma}{\gamma_{\perp}} \sin \varphi} \frac{d y\left(\operatorname{Re} \tau_{+\varkappa}+i y\right)}{1-\varepsilon \cosh \left(\gamma_{\perp} y-i{ }^{\prime} \cos \varphi\right)}$.

The part of the integration that contains $\operatorname{Re} \tau_{+\kappa}$ can be calculated following the procedure described to determine the integral in Eq. (A8). Taking into account Eq. (A9),

$$
\begin{aligned}
\mathcal{J}_{\text {ver }}(\varkappa)^{\varkappa \rightarrow 0} \stackrel{i}{\sim}-\frac{i}{\gamma_{\perp}} \operatorname{Re} \tau_{+\varkappa}(\ln \varkappa+i \varphi) \\
-\frac{1}{\gamma_{\perp}^{2}} \int_{1}^{e^{\gamma \mathrm{cr}+}+\sin \varphi} d s\left[\frac{\ln (s)}{s-s_{+}}-\frac{\ln (s)}{s-s_{-}}\right],
\end{aligned}
$$

where the change of variable $s=\exp \left[\gamma_{\perp} y\right]$ has been used. Here, $s_{+}=\frac{2}{\varepsilon} \exp [i r \cos \varphi]$ and $s_{-}=\frac{\varepsilon}{2} \exp [i r \cos \varphi]$.

The main contribution to the first integral in Eq. (A13) results from $s \sim s_{+}$. Therefore, up to an unessential imaginary term independent of $\gamma$ and $\varphi$, we find

$$
\begin{aligned}
& \frac{1}{\gamma_{\perp}^{2}} \int_{1}^{e^{\gamma \mathrm{cr}+\mu \sin \varphi}} d s \frac{\ln (s)}{s-s_{+}} \\
& \stackrel{r \rightarrow 0}{\approx} \operatorname{Im} \tau_{+\varkappa}\left[\operatorname{Im} \tau_{+\varkappa}+\frac{1}{\gamma_{\perp}}(\ln \gamma+i \varphi)\right] .
\end{aligned}
$$

Conversely, the integration variable linked to the last integral in Eq. (A13) satisfies the condition $s \geq 1 \gg s_{-}$. Hence, by safely ignoring $s_{-}$in the denominator, we end up with

$$
\lim _{\gamma \rightarrow 0} \frac{1}{\gamma_{\perp}^{2}} \int_{1}^{e^{\gamma_{\mathrm{cr}}+r \sin \varphi}} d s \frac{\ln (s)}{s-s_{-}} \approx \frac{1}{2}\left[\operatorname{Im} \tau_{+\ell}\right]^{2}
$$

Combining the outcomes in Eq. (A14) and (A15) into Eq. (A13), we obtain

$$
\begin{aligned}
\mathcal{J}_{\text {ver }}(\varkappa)^{\varkappa \rightarrow 0} & -\frac{1}{\gamma_{\perp}}\left[\operatorname{Im} \tau_{+\ell}+i \operatorname{Re} \tau_{+\ell}\right] \ln \gamma \\
& -\frac{1}{\gamma_{\perp}}\left[\operatorname{Re} \tau_{+\varkappa}+i \operatorname{Im} \tau_{+\ell}\right] \varphi .
\end{aligned}
$$

Inserting this result into Eq. (A5), it is straightforward to verify that the outer integration in Eq. (A3) behaves as

$$
\int_{3 \pi / 2}^{-\pi / 2} d \varphi \ldots \stackrel{r \rightarrow 0}{\sim} \underset{\mathcal{H}^{\frac{2 \epsilon}{\omega}} \operatorname{Re} \tau_{+\hbar}}{ }(\cos \vartheta-i \sin \vartheta)
$$

with $\vartheta=2 \epsilon_{\perp} \omega^{-1} \ln |\gamma| \operatorname{Im} \tau_{+\kappa}$. Therefore, in the limit of $r \rightarrow 0$ and under the condition $\operatorname{Im} \tau_{+k} \gg 1$, the sum over the circles $c_{\varepsilon}$ [see Eq. (A3)] provides no contribution to the single-particle distribution function $W_{T}(\boldsymbol{p})$, either.

\section{APPENDIX B: NO CONTRIBUTION OVER $\mathcal{C}_{ \pm}$}

In this Appendix, we show that the integrals over the vertical segments $\mathcal{C}_{ \pm}$(see Fig. 1) give no contribution in the limit $N \rightarrow \infty$. Let us first denote

$$
I_{ \pm}=\int_{\mathcal{C}_{ \pm}} \frac{d \tau}{1+\tau^{2}} \exp \left[\frac{2 i \epsilon_{\perp}^{2}}{e E_{s}} \int_{0}^{\tau} d \tilde{\tau} f(\tilde{\tau})\right] .
$$

The function contained in the exponent can be found in Eq. (A2). Along $\mathcal{C}_{+}$, the complex integration variable is characterized by $\tau=\pi N / \gamma_{\perp}+i y$. In contrast, over $\mathcal{C}_{-}$the parametrization $\tau=-\pi N / \gamma_{\perp}+i y$ applies. Over the former trajectory, the minimum and maximum values of $y$ are $y_{\min }=0$ and $y_{\max }=\pi N /\left(\sqrt{3} \gamma_{\perp}\right)+1$. Conversely, over the latter, these values exchange their roles. For a very large value of $N \gg 1$,

$$
\begin{aligned}
\left|I_{ \pm}\right|<\frac{\gamma_{\perp}^{2}}{\pi^{2} N^{2}} \int_{0}^{y_{\max }} d y \exp \left[-\frac{\epsilon_{\perp}^{2}}{2 e E_{s}} \operatorname{Im} \mathcal{J}_{ \pm}\right], \\
\mathcal{J}_{ \pm} \equiv \mathcal{J}\left( \pm \frac{\pi N}{\gamma_{\perp}}+i y\right)=\int_{0}^{ \pm \frac{\pi N}{\gamma_{\perp}}+i y} d \tilde{\tau} f(\tilde{\tau}) .
\end{aligned}
$$

In the following, the integrals $\mathcal{J}_{ \pm}$are evaluated by modifying the corresponding paths. However, these deformations depend upon whether the outer integration variable $y$ is smaller or greater than $\operatorname{Im} \tau_{+\kappa}$. Because of this, it turns out to be beneficial to introduce a positive parameter $y_{0} \rightarrow 0^{+}$and split the integral over $y$ accordingly,

$$
\int_{0}^{y_{\max }} d y \ldots=\lim _{y_{0} \rightarrow 0^{+}}\left[\int_{0}^{\operatorname{Im} \tau_{+\hbar}-y_{0}} d y \ldots+\int_{\operatorname{Im} \tau_{+\kappa}+y_{0}}^{y_{\max }} d y \ldots\right] .
$$

Within the sector $\operatorname{Im} \tau_{+\kappa}+y_{0} \leq y \leq y_{\max }$-covered by the second integral in the right-hand side of Eq. (B3) the chosen integration circuit exhibited in Fig. 10 avoids the poles at $\tau_{+\ell}$ with $\ell \in \mathbb{Z}$ [see Eq. (7)]. Conversely, if the outer integration variable lies within $0 \leq y \leq \tau_{+\kappa}-y_{0}$, we can integrate $\mathcal{J}_{ \pm}$-inside the first contribution of the righthand side in Eq. (B3) — via a similar trajectory, with the particularization that its vertical path ends at $y \leq \tau_{+\kappa}-y_{0}$, returning to the origin afterward (dotted line in Fig. 10). As 


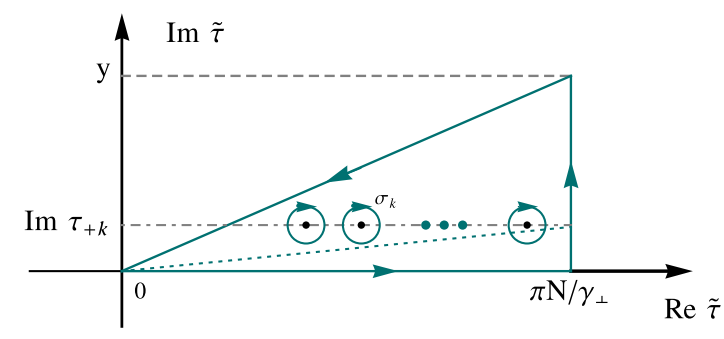

FIG. 10. Integration contours chosen to estimate the behavior of $\mathcal{J}_{+}$[see Eq. (B2)] in terms of the number of cycles $N$. While the thick trajectory is suitable for $y>\operatorname{Im} \tau_{+\kappa}$, the path in dotted style is appropriate if $y<\operatorname{Im} \tau_{+\kappa}$. The corresponding integration circuits linked to $\mathcal{J}_{-}$result from reflections with respect to the imaginary axis $(\operatorname{Re} \tilde{\tau} \rightarrow-\operatorname{Re} \tilde{\tau})$ and by taking a counterclockwise sense. Shortcuts joining each circumventing circle with the real $\tilde{\tau}$ axis have been omitted for simplicity. As in Fig. 1, they give no contribution as both lie infinitesimally close together and have opposite orientation.

all poles are located above the dotted line, no circumvention of them is required in this case.

We will focus on the result coming from the path in which the poles are eluded. However, we will extract parallelly the outcomes linked to the contour in which these circumventions are not necessary. The next step in our analysis is the application of Cauchy's theorem to the problem under consideration. Correspondingly,

$$
\begin{aligned}
\mathcal{J}_{ \pm}= & \int_{0}^{ \pm \frac{\pi N}{\gamma_{\perp}}} d x f(x)+i \int_{0}^{y} d \tilde{y} f\left( \pm \pi N / \gamma_{\perp}+i \tilde{y}\right) \\
& +\sum_{\hbar} \oint_{\sigma_{\hbar}} d \tilde{\tau} f(\tilde{\tau}) .
\end{aligned}
$$

When $0 \leq y \leq \tau_{+\varkappa}-y_{0}$, the expression for $\mathcal{J}_{ \pm}$is only given by the first line of this formula. We note that the integral over the $\operatorname{Re} \tilde{\tau}$ axis is purely real as well as the leading-order contribution of each integration over the small circle $\sigma_{\kappa}\left(\tau=\tau_{+\kappa}+r / \gamma_{\perp} e^{i \chi}\right.$ with $\left.3 \pi / 2 \leq \chi<-\pi / 2\right)$ :

$$
\oint_{\sigma_{\hbar}} d \tilde{\tau} f(\tilde{\tau})^{r \rightarrow 0} \approx \frac{2 \pi}{\gamma_{\perp}}\left(1+\tau_{+\kappa}^{2}\right)^{1 / 2}
$$

Therefore, neither $\int_{0}^{ \pm \frac{\pi N}{\gamma_{\perp}}} d x f(x)$ nor the involved sum contributes to $\operatorname{Im} \mathcal{J}_{ \pm}$, which is precisely what we need [see Eq. (B2)]. In the second integration, we can exploit the condition $\pi N / \gamma_{\perp} \gg 1$ to approximate the multivalued function $\left[1+\left(\pi N / \gamma_{\perp} \pm i y\right)^{2}\right]^{1 / 2} \approx \pi N / \gamma_{\perp} \pm i y$. Consequently,

$$
\begin{aligned}
\operatorname{Im} \mathcal{J}_{ \pm} & \approx \operatorname{Im} i \int_{0}^{y} d \tilde{y} f\left( \pm \pi N / \gamma_{\perp}+i \tilde{y}\right) \\
& \stackrel{N \rightarrow \infty}{\approx} \frac{\pi N}{\gamma_{\perp}} \int_{0}^{y} d \tilde{y} \frac{1}{1+\varepsilon(-1)^{N} \cosh \left(\gamma_{\perp} \tilde{y}\right)} .
\end{aligned}
$$

The integral involved in this expression belongs to the class of integrals treated previously in Appendix A and can be calculated straightforwardly. This leads us to write

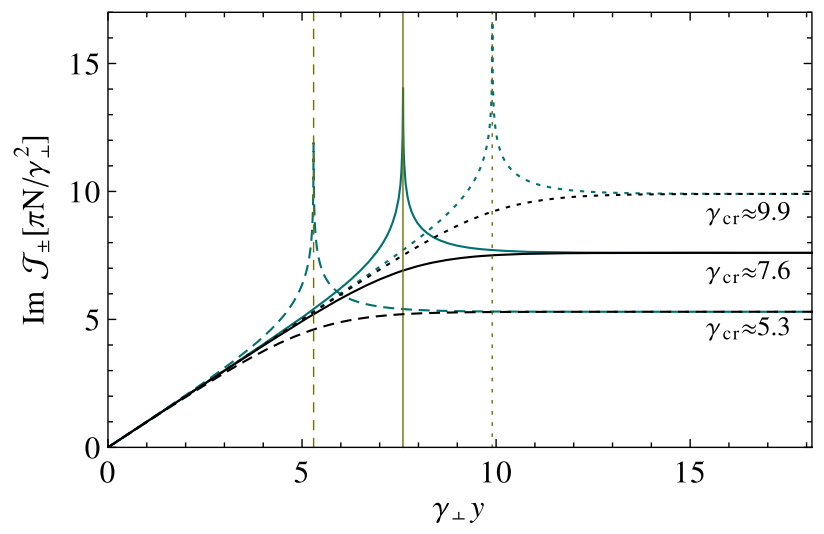

FIG. 11. Behavior of the integral in Eq. (B6). The curves have been obtained for $\varepsilon=10^{-2}$ (dashed), $\varepsilon=10^{-3}$ (solid), and $\varepsilon=$ $10^{-4}$ (dotted). The vertical dashed line in olive depicts the value $\gamma_{\mathrm{cr}} \approx 5.3$, the solid one corresponds to $\gamma_{\mathrm{cr}} \approx 7.6$, whereas $\gamma_{\mathrm{cr}} \approx 9.9$ is shown in dotted style. While the curves in black have been obtained for an even number of cycles, those in darker cyan follow for an odd value of $N$. Each curve manifests a monotonic growth for $0 \leq y \leq \gamma_{\mathrm{cr}} / \gamma_{\perp}$. For $y>\gamma_{\mathrm{cr}} / \gamma_{\perp}$, the curves in darker cyan-corresponding to an odd $N$-fall very fast toward the respective values of $\gamma_{\mathrm{cr}}$. In contrast, each curve in black tends smoothly to the due $\gamma_{\text {cr }}$ parameter.

$$
\operatorname{Im} \mathcal{J}_{ \pm} \approx \frac{\pi N}{\gamma_{\perp}^{2}}\left[y \gamma_{\perp}-\ln \left|1+\frac{\varepsilon}{2}(-1)^{N} e^{\gamma_{\perp} y}\right|\right] .
$$

With this expression at our disposal, the estimation of the integral defined over the sector $0 \leq y \leq \operatorname{Im} \tau_{+\varkappa}-y_{0}$ [see Eq. (B3)] can be carried out without much difficulty. At this point, it is worth mentioning that its integrand has an exponent of which the absolute value grows monotonically with $y$ (see Fig. 11). Hence, the main contribution to $\int_{0}^{\tau_{+\hbar}-y_{0}} d y \ldots$ results from those values $y \ll \operatorname{Im} \tau_{+\varkappa}-y_{0}$. Accordingly, $\operatorname{Im} \mathcal{J}_{ \pm} \approx \pi N y / \gamma_{\perp}$, and

$\lim _{y_{0} \rightarrow 0^{+}} \int_{0}^{\operatorname{Im} \tau_{+\hbar-}-y_{0}} d y \ldots \approx \frac{2 e E_{s}}{\epsilon_{\perp}^{2}} \frac{\gamma_{\perp}}{\pi N}\left[1-\left(\frac{\varepsilon}{2}\right)^{\frac{e E_{s}}{2 \omega^{2}} \pi N}\right]$

Contrary to the previous case, the integral defined over $\operatorname{Im} \tau_{+\varkappa}+y_{0} \leq y \leq y_{\max }$ depends on whether the number of cycles is odd or even. In the former case, the absolute value of the exponent decreases sharply with the increasing of $y$ toward the value $\sim \epsilon_{\perp}^{2} \operatorname{Im} \mathcal{J}_{ \pm} /\left(2 e E_{s}\right)$ with $\operatorname{Im} \mathcal{J}_{ \pm} \approx$ $\pi N \gamma_{\mathrm{cr}} / \gamma_{\perp}^{2}$. However, in the latter situation ( $N$ even), there exists an almost unappreciable growing toward the same value, as the integration variable $y$ increases (see Fig. 11). Hence, the area below both curves approaches

$\lim _{y_{0} \rightarrow 0^{+}} \int_{\operatorname{Im} \tau_{+\kappa}+y_{0}}^{y_{\max }} d y \ldots \approx\left(\frac{\varepsilon}{2}\right)^{\frac{e E_{S}}{2 \omega^{2}} \pi N}\left[\frac{\pi N}{\sqrt{3} \gamma_{\perp}}-\operatorname{Im} \tau_{+\kappa}\right]$ 
Observe that the insertion of Eqs. (B8) and (B9) into Eq. (B3) ensures the convergence to zero of the right-hand side of the first line in Eq. (B2) as $N \rightarrow \infty$. Therefore, no contribution to the single-particle distribution function $W_{T}(\boldsymbol{p})$ results from the integrals over $\mathcal{C}_{ \pm}$. We remark that this statement applies independently of the value of $\operatorname{Im} \tau_{+\kappa}$.
[1] F. Sauter, Über das Verhalten eines Elektrons im homogenen elektrischen Feld nach der relativistischen Theorie Diracs, Z. Phys. 69, 742 (1931).

[2] W. Heisenberg and H. Euler, Folgerungen aus der diracschen theorie des positrons, Z. Phys. 98, 714 (1936).

[3] J. S. Schwinger, On gauge invariance and vacuum polarization, Phys. Rev. 82, 664 (1951).

[4] F. Gelis and N. Tanji, Schwinger mechanism revisited, Prog. Part. Nucl. Phys. 87, 1 (2016).

[5] http://www.eli-laser.eu.

[6] https://xcels.iapras.ru/.

[7] F. Hebenstreit, R. Alkofer, G. Dunne, and H. Gies, Momentum Signatures for Schwinger Pair Production in Short Laser Pulses with Sub-Cycle Structure, Phys. Rev. Lett. 102, 150404 (2009).

[8] S. S. Bulanov, V. D. Mur, N. V. Narozhny, J. Nees, and V. S. Popov, Multiple Colliding Electromagnetic Pulses: A Way to Lower the Threshold of $e^{+} e$ Pair Production from Vacuum, Phys. Rev. Lett. 104, 220404 (2010).

[9] D. B. Blaschke, B. Kämpfer, A. D. Panferov, A. V. Prozorkevich, and S. A. Smolyansky, Influence of laser pulse parameters on the properties of $e^{-} e^{+}$plasma created from vacuum, Contrib. Plasma Phys. 53, 165 (2013).

[10] C. Kohlfürst, M. Mitter, G. von Winckel, F. Hebenstreit, and R. Alkofer, Optimizing the pulse shape for Schwinger pair production, Phys. Rev. D 88, 045028 (2013).

[11] A. Gonoskov, I. Gonoskov, C. Harvey, A. Ilderton, A. Kim, M. Marklund, G. Mourou, and A. Sergeev, Probing Nonperturbative QED with Optimally Focused Laser Pulses, Phys. Rev. Lett. 111, 060404 (2013).

[12] F. Hebenstreit and F. Fillion-Gourdeau, Optimization of Schwinger pair production in colliding laser pulses, Phys. Lett. B 739, 189 (2014).

[13] C. Banerjee, M. P. Singh, and A. M. Fedotov, Phase control of Schwinger pair production by colliding laser pulses, Phys. Rev. A 98, 032121 (2018).

[14] R. Schützhold, H. Gies, and G. Dunne, Dynamically Assisted Schwinger Mechanism, Phys. Rev. Lett. 101, 130404 (2008).

[15] G. Dunne, H. Gies, and R. Schützhold, Catalysis of Schwinger vacuum pair production, Phys. Rev. D 80, 111301 (2009).

[16] D. L. Burke et al., Positron Production in Multiphoton Light-by-Light Scattering, Phys. Rev. Lett. 79, 1626 (1997).

[17] A. Di Piazza, E. Lötstedt, A. I. Milstein, and C. H. Keitel, Barrier Control in Tunneling $e^{+}-e^{-}$Photoproduction, Phys. Rev. Lett. 103, 170403 (2009).

[18] M. J. A. Jansen and C. Müller, Strongly enhanced pair production in combined high- and low-frequency laser fields, Phys. Rev. A 88, 052125 (2013).
[19] S. Augustin and C. Müller, Nonperturbative Bethe-Heitler pair creation in combined high- and low-frequency laser fields, Phys. Lett. B 737, 114 (2014).

[20] S. M. Schmidt, D. Blaschke, G. Röpke, S. A. Smolyansky, and A. V. Prozorkevich, A quantum kinectic equation for particle production in the Schwinger mechanism, Int. J. Mod. Phys. E 07, 709 (1998).

[21] Y. Kluger, E. Mottola, and J. M. Eisenberg, Quantum Vlasov equation and its Markov limit, Phys. Rev. D 58, 125015 (1998).

[22] S. M. Schmidt, D. Blaschke, G. Röpke, A. V. Prozorkevich, S. A. Smolyansky, and V. D. Toneev, Non-Markovian effects in strong-field pair creation, Phys. Rev. D 59, 094005 (1999).

[23] R. Alkofer, M. B. Hecht, C. D. Roberts, S. M. Schmidt, and D. V. Vinnik, Pair Creation and an $X$-ray Free Electron Laser, Phys. Rev. Lett. 87, 193902 (2001).

[24] A. Monin and M. B. Voloshin, Semiclassical calculation of photon-stimulated Schwinger pair creation, Phys. Rev. D 81, 025001 (2010).

[25] M. Orthaber, F. Hebenstreit, and R. Alkofer, Momentum spectra for dynamically assisted Schwinger pair production, Phys. Lett. B 698, 80 (2011).

[26] M. Jiang, W. Su, Z. Q. Lv, X. Lu, Y. J. Li, R. Grobe, and Q. $\mathrm{Su}$, Pair creation enhancement due to combined external fields, Phys. Rev. A 85, 033408 (2012).

[27] I. Akal, S. Villalba-Chávez, and C. Müller, Electronpositron pair production in a bifrequent oscillating electric field, Phys. Rev. D 90, 113004 (2014).

[28] P. Copinger and K. Fukushima, Spatially Assisted Schwinger Mechanism and Magnetic Catalysis, Phys. Rev. Lett. 117, 081603 (2016); Erratum, Phys. Rev. Lett. 118, 099903(E) (2017).

[29] I. A. Aleksandrov, G. Plunien, and V. M. Shabaev, Dynamically assisted Schwinger effect beyond the spatiallyuniform-field approximation, Phys. Rev. D 97, 116001 (2018).

[30] I. Sitiwaldi and B. S. Xie, Pair production by three fields dynamically assisted Schwinger process, Phys. Lett. B 777, 406 (2018).

[31] I. Taya, Franz-Keldysh effect in strong-field QED, Phys. Rev. D 99, 056006 (2019).

[32] K. Krajewska, W. Gac, M. Twardy, and J.Z. Kamiński, Difraction at a time grating in the dynamical sauterschwinger process, J. Phys. 1206, 012018 (2019).

[33] C. Fey and R. Schützhold, Momentum dependence in the dynamically assisted Sauter-Schwinger effect, Phys. Rev. D 85, 025004 (2012).

[34] A. Otto, D. Seipt, D. Blaschke, B. Kämpfer, and S. A. Smolyansky, Lifting shell structures in the dynamically 
assisted Schwinger effect in periodic fields, Phys. Lett. B 740, 335 (2015).

[35] A. Otto, D. Seipt, D. Blaschke, S. A. Smolyansky, and B. Kämpfer, Dynamical Schwinger process in a bifrequent electric field of finite duration: survey on amplification, Phys. Rev. D 91, 105018 (2015).

[36] M. F. Linder, C. Schneider, J. Sicking, N. Szpak, and R. Schützhold, Pulse shape dependence in the dynamically assisted Sauter-Schwinger effect, Phys. Rev. D 92, 085009 (2015).

[37] A. D. Panferov, S. A. Smolyansky, A. Otto, B. Kämpfer, D. B. Blaschke, and Ł. Juchnowski, Assisted dynamical Schwinger effect: Pair production in a pulsed bifrequent field, Eur. Phys. J. D 70, 56 (2016).

[38] C. Schneider and R. Schützhold, Dynamically assisted Sauter-Schwinger effect in inhomogeneous electric fields, J. High Energy Phys. 02 (2016) 164.

[39] C. Schneider and R. Schützhold, Prefactor in the dynamically assisted Sauter-Schwinger effect, Phys. Rev. D 94, 085015 (2016).

[40] G. Torgrimsson, J. Oertel, and R. Schützhold, Doubly assisted Sauter-Schwinger effect, Phys. Rev. D 94, 065035 (2016).

[41] G. Torgrimsson, C. Schneider, and R. Schützhold, SauterSchwinger pair creation dynamically assisted by a plane wave, Phys. Rev. D 97, 096004 (2018).

[42] A. Otto, H. Oppitz, and B. Kämpfer, Assisted vacuum decay by time dependent electric fields, Eur. Phys. J. A 54, 23 (2018).

[43] G. Torgrimsson, Thermally versus dynamically assisted Schwinger pair production, Phys. Rev. D 99, 096007 (2019).

[44] X. G. Huang and H. Taya, Spin-dependent dynamically assisted Schwinger mechanism, Phys. Rev. D 100, 016013 (2019).

[45] G. Torgrimsson, C. Schneider, J. Oertel, and R. Schützhold, Dynamically assisted Sauter-Schwinger effect-nonperturbative versus perturbative aspects, J. High Energy Phys. 06 (2017) 043.

[46] G. Torgrimsson, Perturbative methods for assisted nonperturbative pair production, Phys. Rev. D 99, 096002 (2019).

[47] D. Blaschke, A. V. Prozorkevich, C. D. Roberts, S. M. Schmidt, and S.A. Smolyansky, Pair Production and Optical Lasers, Phys. Rev. Lett. 96, 140402 (2006).

[48] G. Gregori et al., A proposal for testing subcritical vacuum pair production with high power lasers, High Energy Density Phys. 6, 166 (2010).

[49] D. B. Blaschke, G. Ropke, S. M. Schmidt, S. A. Smolyansky, and A. V. Tarakanov, Kinetics of photon radiation off an $e^{-} e^{+}$plasma created from the vacuum in a strong laser field, Contrib. Plasma Phys. 51, 451 (2011).

[50] S. A. Smolyansky, D. B. Blaschke, A. V. Chertilin, G. Roepke, and A. V. Tarakanov, Role of vacuum polarization for the annihilation channel in a strong laser field, arXiv:1012.0559.

[51] D. B. Blaschke, V. V. Dmitriev, G. Ropke, and S. A. Smolyansky, BBGKY kinetic approach for an $e^{-} e^{+} \gamma$ plasma created from the vacuum in a strong laser-generated electric field: The one-photon annihilation channel, Phys. Rev. D 84, 085028 (2011).
[52] F. Karbstein and R. Shaisultanov, Stimulated photon emission from the vacuum, Phys. Rev. D 91, 113002 (2015).

[53] H. Gies, F. Karbstein, and C. Kohlfürst, All-optical signatures of Strong-Field QED in the vacuum emission picture, Phys. Rev. D 97, 036022 (2018).

[54] F. Karbstein, A. Blinne, H. Gies, and M. Zepf, Boosting Quantum Vacuum Signatures by Coherent Harmonic Focusing, Phys. Rev. Lett. 123, 091802 (2019).

[55] A. Blinne, H. Gies, F. Karbstein, C. Kohlfürst, and M. Zepf, All-optical signatures of quantum vacuum nonlinearities in generic laser fields, Phys. Rev. D 99, 016006 (2019).

[56] R. Ruffini, L. Vitagliano, and S. S. Xue, On plasma oscillations in strong electric fields, Phys. Lett. B 559, 12 (2003).

[57] B. Henrich, K. Z. Hatsagortsyan, and C. H. Keitel, Positronium in Intense Laser Fields, Phys. Rev. Lett. 93, 013601 (2004).

[58] A. Ilderton, P. Johansson, and M. Marklund, Pair annihilation in laser pulses: Optical versus $x$-ray free-electron laser regimes, Phys. Rev. A 84, 032119 (2011).

[59] A. I. Voroshilo, S. P. Roshchupkin, and V. N. Nedoreshta, Resonant two-photon annihilation of an electron-positron pair in a pulsed electromagnetic wave, Phys. Rev. A 94, 032128 (2016).

[60] M. G. Mustafa and B. Kämpfer, Gamma flashes from relativistic electron-positron plasma droplets, Phys. Rev. A 79, 020103 (2009).

[61] I. A. Aleksandrov, G. Plunien, and V. M. Shabaev, Photon emission in strong fields beyond the locally-constant field approximation, Phys. Rev. D 100, 116003 (2019).

[62] D. M. Gitman, Processes of arbitrary order in quantum electrodynamics with a pair-creating external field, J. Phys. A 10, 2007 (1977).

[63] E. S. Fradkin, D. M. Gitman, and S. M. Shvartsman, Quantum Electrodynamics with Unstable Vacuum (SpringerVerlag, Berlin, 1991).

[64] C. K. Dumlu, Quantum kinetic approach and the scattering approach to vacuum pair production, Phys. Rev. D 79, 065027 (2009).

[65] C. K. Dumlu and G. V. Dunne, Interference effects in Schwinger vacuum pair production for time-dependent laser pulses, Phys. Rev. D 83, 065028 (2011).

[66] F. Hebenstreit, R. Alkofer, and H. Gies, Schwinger pair production in space- and time-dependent electric fields: Relating the Wigner formalism to quantum kinetic theory, Phys. Rev. D 82, 105026 (2010).

[67] M. Abramowitz and I. Stegun, Handbook of Mathematical Functions (Dover, New York, 1965).

[68] S. P. Kim and D. N. Page, Improved approximation for fermion pair production in inhomogeneous electric field, Phys. Rev. D 75, 045013 (2007).

[69] K. Krajewska and J.Z. Kamiński, Threshold effects in electron-positron pair creation from the vacuum: Stabilization and longitudinal versus transverse momentum sharing, Phys. Rev. A 100, 012104 (2019).

[70] I. Akal and G. G. Moortgat-Pick, Euclidean mirrors: Enhanced vacuum decay from reflected instantons, J. Phys. G 45, 055007 (2018). 
[71] M. J. Ablowitz and A.S. Fokas, Complex Variables Introduction and Applications, 2nd ed. (Cambridge University Press, New York, 2003).

[72] E. Brezin and C. Itzykson, Pair production in vacuum by alternating field, Phys. Rev. D 2, 1191 (1970).

[73] I. S. Gradshteyn and I. M. Ryzhik, Table of Integrals, Series and Products, 7th ed. (Elsevier, San Diego, 2007).

[74] F. W. J. Olver, D. W. Lozier, R. F. Boisvert, and C. W. Clark, NIST Handbook of Mathematical Functions (Cambridge University Press, Cambridge, England, 2010).

[75] V. S. Popov, Resonance pair production in strong electric fields, Pis'ma Zh. Exp. Theor. Fiz. 18, 435 (1973) [JETP Lett. 18, 255 (1973)].

[76] A. Ringwald, Pair production from vacuum at the focus of an $X$-ray free electron laser, Phys. Lett. B 510, 107 (2001).
[77] V.S. Popov, The Schwinger effect and possibilities for its observation using optical and $X$-ray lasers, Sov. Phys. JETP 94, 1057 (2002).

[78] M. Ruf, G. R. Mocken, C. Müller, K. Z. Hatsagortsyan, and C. H. Keitel, Pair Production in Laser Fields Oscillating in Space and Time, Phys. Rev. Lett. 102, 080402 (2009).

[79] I. A. Aleksandrov, G. Plunien, and V. M. Shabaev, Momentum distribution of particles created in space-time-dependent colliding laser pulses, Phys. Rev. D 96, 076006 (2017).

[80] Q.Z. Lv, S. Dong, Y. T. Li, Z. M. Sheng, Q. Su, and R. Grobe, Role of the spatial inhomogeneity on the laser-induced vacuum decay, Phys. Rev. A 97, 022515 (2018).

[81] W. Greiner and J. Reinhart, Quantum Electrodynamics, 3rd ed. (Springer, Heidelberg, 2003). 